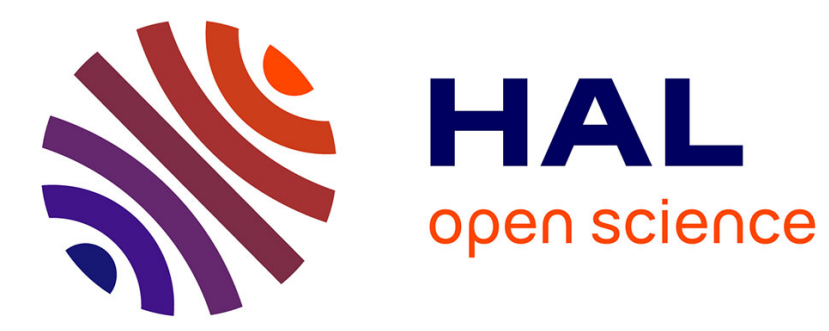

\title{
Brightness power spectral distribution and waves in Jupiter's upper cloud and hazes
}

\author{
N. Barrado-Izagirre, S. Pérez-Hoyos, A. Sánchez-Lavega
}

\section{To cite this version:}

N. Barrado-Izagirre, S. Pérez-Hoyos, A. Sánchez-Lavega. Brightness power spectral distribution and waves in Jupiter's upper cloud and hazes. Icarus, 2009, 202 (1), pp.181. 10.1016/j.icarus.2009.02.015 . hal-00545288

\section{HAL Id: hal-00545288 \\ https://hal.science/hal-00545288}

Submitted on 10 Dec 2010

HAL is a multi-disciplinary open access archive for the deposit and dissemination of scientific research documents, whether they are published or not. The documents may come from teaching and research institutions in France or abroad, or from public or private research centers.
L'archive ouverte pluridisciplinaire HAL, est destinée au dépôt et à la diffusion de documents scientifiques de niveau recherche, publiés ou non, émanant des établissements d'enseignement et de recherche français ou étrangers, des laboratoires publics ou privés. 


\section{Accepted Manuscript}

Brightness power spectral distribution and waves in Jupiter's upper cloud and hazes

N. Barrado-Izagirre, S. Pérez-Hoyos, A. Sánchez-Lavega

PII: S0019-1035(09)00072-4

DOI: $\quad$ 10.1016/j.icarus.2009.02.015

Reference: $\quad$ YICAR 8925

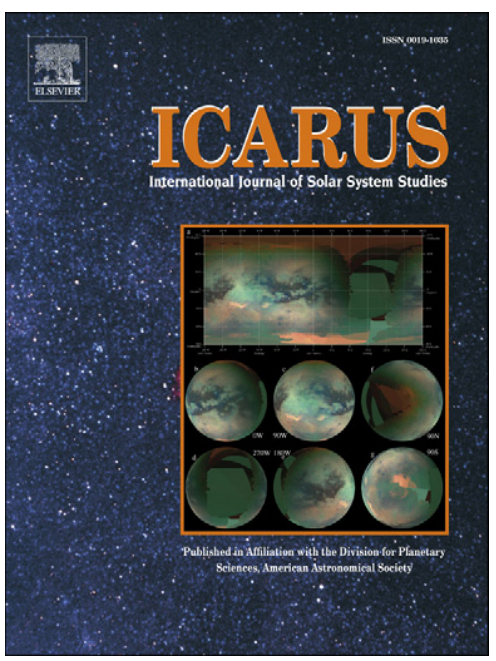

To appear in: Icarus

Received date: 30 May 2008

Revised date: 6 February 2009

Accepted date: 13 February 2009

Please cite this article as: N. Barrado-Izagirre, S. Pérez-Hoyos, A. Sánchez-Lavega, Brightness power spectral distribution and waves in Jupiter's upper cloud and hazes, Icarus (2009), doi:

10.1016/j.icarus.2009.02.015

This is a PDF file of an unedited manuscript that has been accepted for publication. As a service to our customers we are providing this early version of the manuscript. The manuscript will undergo copyediting, typesetting, and review of the resulting proof before it is published in its final form. Please note that during the production process errors may be discovered which could affect the content, and all legal disclaimers that apply to the journal pertain. 


\section{Brightness power spectral distribution and waves in Jupiter's upper cloud and hazes}

N. Barrado-Izagirre ${ }^{a, *}$, S. Pérez-Hoyos ${ }^{a}$, A. Sánchez-Lavega ${ }^{a}$

${ }^{\text {a }}$ Grupo de Ciencias Planetarias,

Dpto. Física Aplicada I, Escuela Superior de Ingenieros,

Universidad del País Vasco,

Alda. Urkijo s/n, 48013 Bilbao (Spain)

* E-mail: naiara.barrado@ehu.es

Manuscript Pages $=50$

Tables $=5$

Figures $=8$ 
Proposed running head: Jupiter's brightness spectral distribution

\section{Editorial correspondence to:}

Naiara Barrado-Izagirre

Departamento Física Aplicada I

Escuela Superior de Ingeniería

Universidad del País Vasco

Alda.Urkijo s/n

48013 Bilbao (Spain)

Telephone: (34) 94.601.39.97

Fax: (34) 94.601.41.78

e-mail: naiara.barrado@ehu.es 


\section{Abstract}

In this work we analyze the spatial structure of Jupiter's cloud reflectivity field in order to determine brightness periodicities and power spectra characteristics together with their relationship with Jupiter's dynamics and turbulence. The research is based on images obtained in the near-infrared $(\sim 950 \mathrm{~nm})$, blue $(\sim 430 \mathrm{~nm})$ and near-ultraviolet $(\sim 260 \mathrm{~nm})$ wavelengths with the Hubble Space Telescope in 1995 and the Cassini spacecraft Imaging Science Subsystem in 2000. Zonal reflectivity scans were analyzed by means of spatial periodograms and power spectra. The periodograms have been used to search for waves as a function of latitude. We present the values of the dominant wavenumbers for latitude bands between $32^{\circ} \mathrm{N}$ and $42^{\circ} \mathrm{S}$. The brightness power spectra analysis has been performed in the meridional and zonal directions. The meridional analysis of albedo profiles are close to a $k^{-5}$ law similarly to the wind profiles at blue and infrared wavelengths, although results differ from that in the ultraviolet. The zonal albedo analysis results in two distributions characterized by different slopes. In the near infrared and blue wavelengths, average spectral slopes are $n_{1}=-1.3 \pm 0.4$ for shorter wavenumbers $(k<80)$, and $n_{2}=-2.5 \pm 0.7$ for greater wavenumbers, whereas for the ultraviolet $n_{1}=-1.9 \pm 0.4$ and $n_{2}=-0.7 \pm 0.4$, possibly showing a different dynamical regime. We find a turning point in the spectra between both regimes at wavenumber $k \sim$ 80 (corresponding to $L \sim 1000 \mathrm{~km}$ ) for all wavelengths.

Key Words: Jupiter, atmosphere; Atmospheres, dynamics; Atmospheres, structure. 


\section{Introduction}

Jupiter's visual appearance is characterized by a pattern of bright zones and dark belts in alternating latitudinal bands. This banded structure is correlated with a zonal circulation dominated by a system of jets, the zones corresponding to anticyclonic vorticity areas and belts to cyclonic vorticity regions (Ingersoll et al. 2004; Vasavada and Showman 2005). A variety of dynamical phenomena inside the belt/zone structure is detected by their cloud patterns (waves, irregular storms, closed vortices, etc.). The zonal banding brightness is far from being homogeneous, when observed at high resolution, showing the existence of chaotic (turbulent) areas, sometimes spatially organized in particular in the strongly meridionally sheared regions. Some examples are, the Great Red Spot wake (westward), the cyclonic filamentary regions (Mitchell 1982) or others that sporadically form when large scale disturbances occur, as during the South Equatorial Belt events (Sánchez-Lavega and Gomez 1996) or the North Temperate Belt disturbances (e.g. see Sánchez-Lavega et al. 2008). In fact, Jupiter’s visible clouds represent a privileged site for the study of chaotic and turbulent cloud patterns.

One strategy to characterize the global cloud patterns in planetary atmospheres is the analysis at different wavelengths of the cloud field brightness, as Travis (1978) did for the case of Earth and Venus, Peralta et al. (2007) for Venus and Harrington et al. (1996a, 1996b) for Jupiter. The brightness structure is important for two reasons. First, we can determine the clouds' size and shape distribution, together with their periodicity (if any). Since cloud morphology (organization and distribution) is linked to the dynamical mechanisms operating at a global scale, its study is a proxy for the dynamical phenomena. The second reason is the connection between the brightness power spectra and the kinetic energy power spectra first suggested by Travis (1978) for the Earth case. 
Jupiter is especially well suited for the brightness spectrum approach to the kinetic energy spectrum because of the correlation between the banded clouds and the zonal jet system stated above.

However, atmospheric turbulence is not well constrained in most of the planetary atmospheres, with the possible exception of the Earth (Nastrom et al. 1984; Nastrom and Gage, 1985). Turbulence has been addressed as a main tool for understanding the atmospheric dynamical mechanisms (see e.g. Vallis 2006). It affects many dynamical variables (e.g. pressure, temperature, wind speed) but its characterization is not simple since a large range of spatial and temporal scales are involved. On rapidly rotating planets, like Jupiter, the wind system outside the Equator is under quasi-geostrophic equilibrium (Ingersoll et al. 2004). The theoretical study of the quasi-geostrophic turbulence in a sphere (Rhines 1975) combined with numerical studies with or without forcing and dissipation (Williams, 1978, 1979; Cho and Polvani, 1996; Scott et al. 2007), showed that zonal winds could result from the combination of planetary rotation with the so-called inverse cascade of energy, characteristic of twodimensional turbulence (Vallis 2006). The measurement of turbulent motions (turbulent wind speeds, $\left.u^{\prime}, v^{\prime}\right)$ is not an easy task since typical values are similar to the standard measurement errors. The global turbulent zonal velocity field $\left(u^{\prime}, v^{\prime}\right)$ and the resulting spectral energy distribution $E_{K}^{\prime}$ and averaged eddy fluxes $\left\langle u\right.$ ' $\left.v^{\prime}\right\rangle$ were measured in Jupiter using Voyager images (Beebe et al. 1980 and Ingersoll et al. 1981) although they were questioned later by Sromovsky et al. (1982). Recently, using Cassini images, eddy motions were measured in some Jupiter (Salyk et al. 2006) and Saturn (Del Genio et al. 2007) areas, confirming the momentum transfer from the eddies to the eastward jets and the energy conversion rates derived from the Voyagers' analysis. The only published studies of the power spectral laws for the turbulent zonal kinetic energy in 
Jupiter were done by Mitchell (1982) and Mitchell and Maxworthy (1985), based on the analysis of Voyager observations.

Because of the inhomogeneous distribution of the upper aerosols (clouds and hazes) in Jupiter's atmosphere, the first step is to locate vertically the altitude of the clouds sensed at different wavelengths. Most of the visible light comes from pressure levels between 250 mbar and 1 bar where the ammonia cloud base is approximately located (West et al. 2004). At shorter wavelengths, in the near ultraviolet, we observe the upper hazes above these clouds. Here, the zonal winds decrease their intensity (Gierasch et al. 1986, Flasar et al. 2004, Li et al. 2006a) and an extremely different horizontal cloud structure is observed. On the other hand, near infrared wavelengths (from $800 \mathrm{~nm}$ to $1 \mu \mathrm{m}$ ) outside the intense methane absorption bands sound deeper into the ammonia cloud. Therefore, we study the reflectivity structure at three wavelengths that sense the lower stratosphere $(<100$ mbar, near ultraviolet observations), the upper troposphere $(200-700$ mbar, blue images) and the ammonia cloud level $(800-1000$ mbar, near infrared), approximately.

The aim of this paper is basically two-fold. First, we search for wave phenomena at those cloud levels using Lomb-Scargle periodograms (a normalized Fast Fourier Transform) analysis in order to determine their basic properties. Second, we study the brightness distribution power spectra and compare it with that expected from the laws of the turbulence theory. The cloud brightness distribution and waves are finally studied in the context of Jupiter's zonal wind and vorticity profiles. We have used images from Hubble Space Telescope (HST) obtained in 1995 and from Cassini spacecraft obtained during its fly-by of Jupiter in 2000 (Porco et al. 2003). Therefore, the cloud brightness pattern is compared at two different epochs, searching for temporal changes and evolution of the observed patterns. 
This work is divided in five sections. First, we present in Section 2 a detailed explanation of the observations (filters, dates and resolution); this section also deals with image navigation and processing in order to compose planispheres. In Section 3 we describe the methodology used for the systematic search of atmospheric waves as well as an explanation on the retrieval of brightness power spectra from the map projections, showing some representative examples. In section 4 , we summarize our main results which are discussed in section 5 in terms of models and previous turbulence results as well as a summary of our conclusions.

\section{Observations}

The Wide Field Planetary Camera 2 (WFPC2) onboard the HST and the Cassini Imaging Science Subsystem (ISS) observations used in this work correspond to filters centred at ultraviolet, blue and near infrared wavelengths (see Table 1 for details). As explained above, we sound in the ultraviolet $(260 \mathrm{~nm})$ the homogeneous higher hazes. Observations at $950 \mathrm{~nm}$ (near infrared) show the ammonia cloud deck, very rich in detail. Finally, the $430 \mathrm{~nm}$ images (blue) present the highest contrast corresponding to intermediate levels. Details on the Cassini filters can be found in Porco et al. (2004) and for the HST filters in Pérez-Hoyos et al. (2005), and references therein. Both sets of filters are compared in Sánchez-Lavega et al. (2006).

\section{[Table 1]}

In order to perform a full zonal wave search and power spectral analysis we need to build a complete planisphere (cylindrical map projection). For obtaining so, we selected images containing a full Jupiter's disk, if possible. Typically, at least six images were required to cover all the longitudes, with their central meridian separated by about $60^{\circ}$ and a time span of about 1 hour and a half, avoiding the typical temporal 
evolution of the features, especially at the smaller spatial scales. The latitudinal coverage was constrained by these factors together with the viewing geometry.

There are a few differences between the HST and Cassini sets of images. The first one is the spatial resolution. Although there are higher resolution images in the Cassini ISS archive, the selected images are best suited for the planisphere composing (following the previous paragraph criteria) and accommodate to the same spectral range as available for HST observations. HST images have a slightly higher resolution $(0.15$ o/pix $\left.=187 \mathrm{~km} \mathrm{pix}^{-1}\right)$ than the Cassini ones $\left(0.213 \%\right.$ pix $\left.=265 \mathrm{~km} \mathrm{pix}^{-1}\right)$. The second difference is the viewing geometry, with planetocentric sub-solar latitudes of $-2.6^{\circ}$ for HST and $3.7^{\circ}$ for Cassini; the only consequence of this is that the planispheres have a slightly different latitudinal coverage.

We retrieved both data sets and their corresponding navigation and ancillary data from NASA's Planetary Data System. In the case of Cassini images, selection was made through PVOL software (Morgado et al. 2005). HST images were pipeline preprocessed and calibrated and Cassini ISS ones were photometrically calibrated with CISSCAL software (Porco et al. 2004). We used LAIA software (Cano, 1998; see e. g. García-Melendo and Sánchez-Lavega, 2001 for details) for HST image navigation and projection; whereas for Cassini images PLIA software was employed (Hueso et al. 2008). Both use parts of the VICAR code for pixel to longitude-latitude coordinates transformation (Barrey 1984). Limb-darkening was corrected applying Minnaert's law (Minnaert 1941):

$(I / F)=(I / F)_{0} \cdot \mu^{k-1} \cdot \mu_{0}^{k}$

where $(I / F)_{0}$ is the planisphere's corrected reflectivity; $(I / F)$ is the observed reflectivity; $k$ is the limb-darkening coefficient and $\mu$ and $\mu_{0}$ are the cosines of the viewing and illuminating angles that are given by Horak (1950): 
$\mu=\sin B \cdot \sin \varphi+\cos B \cos \varphi \cos (\Delta L)$

$\mu_{0}=\sin B^{\prime} \cdot \sin \varphi+\cos B^{\prime} \cos \varphi \cos (\Delta L-\alpha)$

$B$ is the sub-observer planetocentric latitude, $B^{\prime}$ is the sub-solar planetocentric latitude, $\alpha$ is the phase angle, $\varphi$ is the planetocentric latitude and $\Delta L$ the longitude from central meridian.

In order to retrieve the limb-darkening coefficient $k$ at each latitude in a given planisphere we expressed the Minnaert's law logarithmically:

$\ln (\mu I / F)=k \cdot \ln \left(\mu \mu_{0}\right)+\ln \left(\mu I / F_{0}\right)$

Fitting $\ln (\mu I / F)$ versus $\ln \left(\mu \mu_{0}\right)$ we retrieve the slope $k$ through a least-squares linear fit. In such a way, we obtained $k(\varphi)$ for each planisphere to correct it from limbdarkening.

Finally, in order to obtain smooth planispheres we have to pay attention to overlapping regions (i.e. regions shared by two images). We gave a specific weight to each pixel depending on the distance to the overlapping edge. Thus, to estimate image value at overlapping pixels we used the following expression (Peralta et al. 2007):

$R^{i, j}=\frac{R_{1}^{i, j}\left(i_{2}-i\right)+R_{2}^{i, j}\left(i-i_{1}\right)}{i_{2}-i_{1}}$

where $R_{1}, R_{2}$ and $R$ are the brightness of the image located on the left, the second image located on the right and resulting planisphere, respectively. The indexes $i$ and $j$ correspond to the horizontal and vertical pixel respectively, whereas $i_{1}$ and $i_{2}$ are the right and left position of the overlapping edge. In this way, we get a smooth transition between individual planispheres as shown in Fig. 1a-c (left panels).

\section{[Figure 1]}




\section{Analysis of the spatial brightness distribution}

The Fast Fourier Transform (FFT) is used in this work not only to investigate the contribution of each wavenumber to the observed reflectivity field but also to determine the characteristic frequency of periodic structures. Planispheres were over-sampled to $0.1 \%$ pix resolution in both the zonal and meridional direction. On each of the six planispheres shown in Fig. 1, we performed zonal brightness scans across latitude circles. It is also possible to use more sophisticated strategies such as the projection onto spherical harmonics using decomposition into Legendre polynomials. This is more accurate but computationally slower, so we have checked that our approach is compatible with this one for some selected situations. To determine the spatial scales of the brightness variability we used, instead of frequency or wavelength, the nondimensional zonal wavenumber $k$ :

$$
k=2 \pi R(\varphi) \cos (\varphi) / L
$$

where $R(\varphi)$ is the planetary radius, $\varphi$ is the planetocentric latitude and $L$ is the spatial wavelength. Therefore, $k$ represents the number of waves that fits the whole longitude circle.

Figure 2 shows the FFT power spectrum on a latitude band, that corresponds to the location of the Hot Spots dark projections area at $7^{\circ} \mathrm{N}$ which is a well known wavy region at visual wavelengths (Arregi et al. 2006). The Fast Fourier Transform of a given signal transforms the input from the space domain to the frequency domain. This is in general a complex function although in this work we will focus on the squared amplitude retrieved multiplying the FFT by its complex conjugate, as usually done in this kind of studies (Brigham 1988). So, in the end, we retrieve the squared amplitude contribution of each frequency to the observed intensity.

[Figure 2] 


\subsection{Periodic brightness structures}

The Fast Fourier transform in a linear scale with Lomb-Scargle normalization (Lomb 1976, Scargle 1982), also known as periodogram, is especially well suited for the study of characteristic wavenumbers of strongly contrasted regularly spaced albedo structures. This way of representation is more convenient than the logarithmic for the study of atmospheric waves' periodicity and their latitude location and extent because of its normalization that can be related to the statistical significance of each peak. In Figs. 2a-c we show the cloud field maps (left panels) together with the corresponding brightness spectral maps (right) up to wavenumber 20 for both epochs 1995 and 2000. Since a given periodic feature exhibits various degrees of contrast along its crests and valleys, maps show not only a single characteristic wavenumber but a combination of the most strongly contributing harmonics. The power value can be related to the statistical significance of the periodicity by means of white noise simulations which throw the probability that a given peak strength is produced by noise (e.g., see Fletcher et al. 2008).

\subsection{Brightness Power Spectral Laws}

The FFT tells us how each wavenumber contributes to the observed intensity and gives information on how such brightness is distributed among all spatial scales. This is approximated by a power law function $P(k)=P_{0} k^{-n}$, with power spectrum slope $n$.

There are some issues that should be taken into account when analyzing power spectra. Firstly, the digitalization of a signal limits the dynamical range of the data to a finite number of intensity levels, so its power spectrum is going to be influenced by the 
signal quantification (Peralta et al. 2007). Our planispheres have a dynamical range between 8 and 12 bits (this dynamical range is caused not only by the a priori capability of the camera, but also by the subexposure of each individual image, which confine the available information to a limited number of bits). Using tests of a sinusoidal signal with expected slopes of the power spectrum (from -1 to -5) and different spatial resolutions, we find that digitalization imposes an upper wavenumber limit of $k_{d}=150$ for the worst case (slope of -3.5 and spatial resolution of $0.5^{\circ}$ pix $^{-1}$ ). Another problem is the aliasing effect introduced by the finite spatial resolution which produces a spurious power at all wavenumbers becoming dominant over the real signal as $k$ increases. This effect can be tested transforming a synthetic signal into a number of spatial resolutions (Peralta et al. 2007). It is limited by the Nyquist frequency which can be estimated as $k_{N}$ $=\left(360^{\circ} \cdot \cos \varphi\right) /(E \cdot F)$ where $E$ is the resolution of the image and $F$ is a factor accounting for the minimum detectable extent of a feature (ideally, $F=1$ pixel). In our case $k_{N}=$ 400, greater than the digitalization maximum wave number, which is taken as the limiting factor. These limiting factors are found to depend on the wavelength of the observations (e.g. ultraviolet observations show a smaller dynamical range) but we always restrict as much as possible to wavenumbers where this effect is negligible.

On the other hand, we fixed a minimum wavenumber (corresponding to longer scale details) from which to start the power spectra slope measurement. This point is more difficult to constrain, since there is no a priori analytical convention, nor a physical reason except for the range of Rossby waves propagation (Mitchell 1982). In this work, we assumed the minimum wavenumber to be $k_{m}=10$, in order to avoid the effect that easily observable features (such as the Great Red Spot, the White Ovals or the Hot Spots) may introduce in the retrieved slopes of the power spectra. In fact, the 
contribution of $\mathrm{k}=1-10$ turned out to be negligible for our purposes as it will be explained later.

\section{[Figure 3]}

At first look, most of the power spectra show two different slopes for ranges corresponding to lower and higher zonal wavenumbers (Fig. 3; see also Harrington et al. 1996b). Those ranges are separated by a turning point at wavenumber $k_{I}$. In order to find the best fit to one or two straight lines and the corresponding turning point we automatically scanned all possible turning points' position and fits lines. We computed the root mean square (rms) of each fit selecting as result the one with the minimum error. For most cases, the power spectra are best fitted by two slopes being the turning point at about $k_{I} \sim 80$. Figure 3 shows representative cases of the brightness scan and FFT analysis for selected Jovian latitudes. The presence of the vortices on the brightness profile strongly influences the power spectra slopes. They are present not only at the lower wavenumbers corresponding to their characteristic size but also at higher wavenumbers. This means that at some latitudes the slopes contain information and are modulated by the presence of detached brightness features rather than solely that of the small scale turbulence. Results corresponding to such locations should be taken with care when relating brightness power spectra to atmospheric dynamics.

\section{Results}

\subsection{Wave search}

Our periodogram analysis is shown as maps in Fig. 1 (lower wavenumbers, $k=$ 3 -20). Higher wavenumbers have been also investigated, although they are not shown here for simplicity. Each map shows the power as a function of latitude and wavenumber for HST (1995) and Cassini (2000) epochs at the three selected filters. 
The brightness code in the map indicates the latitude location of the dominant wave numbers (highest powers) for all the periodic brightness structures that appear in each planisphere. Table 2 summarizes the location and wavenumbers of the periodic structures observed in the near infrared images most likely to be related with atmospheric waves, selected through visual inspection. Precise determination of characteristic wavenumber for each case requires a more detailed analysis (higher temporal resolution and adequate image processing for the particular region).

\section{[Table 2]}

Different kind of structures produces periodic patterns: (1) anticyclonic vortices chains, (2) undulating albedo structures (for example, at belt-zone boundaries), (3) bright and dark albedo patches (as the Hot Spots visible counterparts and related projections), (4) turbulent and chaotic patterns (as in the GRS wake), and (5) cyclonic filamentary areas. Although each morphological cloud structure corresponds to a different dynamical phenomenon, all of them produce periodic brightness patterns.

The blue maps (Fig. 1b) show periodicities at almost the same latitudes as the near infrared ones. The ultraviolet maps (Fig. 1c) are flat and homogeneous in albedo and therefore show less wave activity. However, some striking features arise at the ultraviolet maps, as for example, the polar wave at $57^{\circ} \mathrm{S}$ in 1995 and 2000 (Vincent et al. 2000; Barrado-Izagirre et al. 2008). Particularly interesting is the wave patchy train at $15^{\circ} \mathrm{N}$ observed during the Cassini period (discovery by Li et al. 2006b) that was not present, however, in the HST maps. This kind of changes between 1995 and 2000 can be noted at many latitudes, without significant changes in the zonal winds. As an example a notorious variation between both epochs occurred at $33^{\circ} \mathrm{S}$ following the three consecutive merges of the anticyclones known as the White Oval Spots (SánchezLavega et al. 2000, 2001). This is apparent in the ultraviolet periodograms which 
clearly show the vortex chain in 1995 (with characteristic wavenumbers of about $\sim 12$ ) but not in 2000 when only one white oval survived, designated BA.

\subsection{Winds and brightness meridional profile power spectra}

Galperin et al. (2001) and Sukoriansky et al. (2002) presented a power spectrum analysis of the zonal jet system meridional profile for all the giant planets. Their results indicate for all cases a dependence of the zonal energy per unit wave number following a power-law $\sim k^{-5}$ with an amplitude value related to the planetary radius $(R)$ and the rotation rate $(\Omega)$ in the following way:

$$
E_{z}(k)=C_{Z}(\Omega / R)^{2} k^{-5}
$$

Where $C_{Z}$ is a constant expected to be around $C_{Z}=0.3-0.5$ (Galperin et al. 2001). This law holds for a given range of zonal wavenumbers. It should be noted that in this sub-section $k$ is a meridional wave number, similarly to the zonal wavenumber defined in Eq. (6). This law was theoretically predicted for two-dimensional anisotropic turbulence on the surface of a rotating sphere under the $\beta$-effect by Huang et al. (2001).

This behavior was proposed to occur in Earth's oceans (Galperin et al. 2004) and observed in tank laboratory measurements (Read et al. 2007). This law differs from those predicted by two-dimensional turbulence (Kolmogorov's spectrum, $E(k) \sim k^{-5 / 3}$ ) in the inertial scale range and from the $E(k) \sim k^{-3}$ spectrum for larger wavenumbers (Vallis 2006). Our first FFT power spectra analysis was focused on the wind meridional structure to confirm this result but also to extend it in time and wavelength. In addition to the zonal winds, we also performed an analysis of the reflectivity meridional profiles. A comparison of the amplitude of the brightness and zonal wind speed spectra is not straightforward and it will not be discussed here.

\section{[Figure 4]}




\section{Zonal winds}

Galperin et al. (2001) studied the wind profile measured by Simon (1999) obtaining a slope of -4.1 for the meridional wavenumber range $20<k<90$. They also found that for the wavenumbers in which the slope tends to $\sim-5$ the constant $C_{Z}$ was also in the expected range $0.3-0-5$. That wind profile was obtained from a reanalysis of Voyager images obtained in 1979 at green wavelengths. Similarly, Sukoriansky et al. (2002) analyzed the HST profile for 1995 measured by García-Melendo and SánchezLavega (2001) in the near infrared $(\sim 950 \mathrm{~nm})$ and stated the slope to be close to the predicted value of -5 . This is the same wind profile we used for HST (results summarized in Table 3). For the wavenumber range $20<k<90$ (selected for comparison with cited works) we retrieve a slope value of -4.1 and for $20<k<70 C_{Z} \sim 0.3$, as found by Galperin et al. (2001) for Voyagers winds. In order to extend the temporal coverage, we analyzed similarly the 2000 Cassini profile measured by Porco et al. (2003) in the near infrared wavelengths, obtaining a slope of -4.6 that falls within the error bars of previous results (Fig. 4a). Similarly, we also find $C_{Z} \sim 0.5$, as predicted. All the wind profiles presented so far correspond to the pressure range between 600 mbar and 1 bar. In order to complete these results, we have also analyzed the winds measured by cloud tracking on Cassini ultraviolet images by Li et al. (2006a), placed at a higher atmospheric level (close to the tropopause at 100 mbar). This profile is different from that observed at longer wavelengths, with less intense peak values. This results in a power spectrum slope of -2.5 (Fig. 4b), which is substantially lower than previous slopes. This significant decrease for higher altitude winds suggests that the $k^{-5}$ law does not hold for this case. The corresponding $C_{Z}$ value is also substantially lower than that 
retrieved for deeper penetrating observations. This points towards a different dynamical behavior at higher altitude levels that merits future research.

\section{Cloud Reflectivity}

The meridional cloud reflectivity profiles and their power spectra analysis are presented in Fig. 4c and 4d for the near infrared and ultraviolet in Cassini epoch and Table 3 summarizes results for both epochs in the three wavelengths. Two aspects stand out: first, the slopes are similar for both periods at each wavelength; and second, there is a good correlation between slopes obtained for wind speed and those retrieved from reflectivity meridional profiles as shown in Table 3 . This is an indication of the good correlation between the zonal wind pattern and the belt/zone cloud structure, even at

higher atmospheric levels and encourages a regional (zonal) study of the cloud brightness power spectra, and its comparison with the above meridional profiles (wind speed and reflectivity).

\section{[Table 3]}

\subsection{Zonal power spectra}

A zonal brightness scan was performed in steps of $0.1^{\circ}$ of latitude for each period (1995 and 2000) and wavelength (ultraviolet, blue and near infrared) with a power spectrum being obtained from each individual scan. Most spectra have two slopes $n_{1}$ and $n_{2}$ separated at a turning point $k_{I}$. In order to retrieve the meridional behaviour of the zonal power spectra slopes and to reduce the spectra noise, we averaged them on meridional domains attending to the following criteria: (1) Eastward and westward jets as limits of the domain. (2) Latitude bands with the same vorticity 
sign (cyclonic and anticyclonic regions). (3) Albedo bands (belts and zones). The results are plotted in Figs. 5-7 in a similar way.

[Figure 5]

[Figure 6]

[Figure 7]

Figures 5, 6 and 7 show the panels corresponding to the near-infrared, blue and ultraviolet wavelength analysis following the integration criteria presented above. In Table 4 we summarize the results for the averaged slopes and position of the turning point for the different criteria and wavelengths. The most obvious result from this Table 4 is that the slope $n_{1} \sim-1.3$ (on average, excluding the ultraviolet results) is far away from the slopes derived in the previous meridional analysis. Something similar happens for $n_{2}$ where mean value is $n_{2} \sim-2.5$.

\section{[Table 4]}

In order to understand the significance of these results, we discuss the dependence with wavelength and the correlation with physical variables (winds, shear and brightness) in the following paragraphs.

\section{Wavelength variability}

Firstly, within each wavelength, the slopes have a homogeneous behavior independently of the selected integration criterion that depends on the latitude. A similar behavior is found for the turning point separating both slopes. This clearly indicates that the averaged power spectra distribution is strongly controlled by wavelength not by the selected domain. Secondly, we see for $n_{l}$ that the HST data show a significant wavelength dependence ( -2.4 at ultraviolet to -1.3 at near infrared) whereas the Cassini set shows no noteworthy wavelength variation for $n_{1}$ with a mean value of -1.3 (near 
infrared, blue and ultraviolet). For $n_{2}$, a significant wavelength dependence is found (0.6 at ultraviolet but -2.5 at blue and near infrared) and it is similar for both HST and Cassini data sets.

Correlations with dynamics and global albedo

Although the latitudinal dependence of the slopes was found to be very consistent in both blue and infrared observations for 1995 and 2000, we find no obvious correlation between wind speed and its meridional shear with the brightness structure. No correlation is found between the retrieved slopes of the brightness power spectra and the jets speed and ambient flow vorticity.

Finally, we note that the latitudinal behavior of the $n_{1}$ slope profiles at the near infrared suggests an increasing trend from $\sim-2.5$ at equator to $\sim-0.75$ at $60^{\circ}$ latitude, with a less pronounced but similar tendency at blue wavelengths. This meridional behavior occurs for any type of magnitude considered (winds, shear or albedo) so it could be related to dependence with latitude of the Coriolis parameter $(f)$ or its meridional derivative $(\beta)$ as suggested by quasi-geostrophic models (Williams 1978, 1979). The absolute value of the former is positively correlated with $n_{l}$ whereas the later would be anticorrelated. However, a theory linking those parameters with the turbulence is still to be developed.

\section{Discussion of the zonal power spectra and conclusions}

The cloud albedo power spectra presented in section 4 suggest an interpretation in terms of the turbulence theories. The classical 2D- and 3D- turbulence theories for the kinetic energy power spectra (Kolmogorov 1941; Kraichnan 1967; see e.g. Vallis 2006, for a comprehensive review) predict different values for the slopes of the power 
laws depending on the wavenumber and operating turbulent regime. In the $3 \mathrm{D}$ turbulent regime there is only one slope-range, the direct energy cascade where the energy is transferred from large to smaller scales, with a slope of $n=-5 / 3=-1.66\left(k^{-5 / 3}\right.$ law). For the 2D-turbulent regime there are two different ranges: an inverse energy cascade (energy transfer from small to large scales) with the same slope as in the 3D-case and the enstrophy cascade (direct enstrophy transfer) with $n=-3$ slope ( $k^{-3}$ law). The limit between both ranges occurs at the spatial scale of the characteristic dynamical forcing of the flow. Another point of view for this problem is based on the passive tracers' theory. Spectra may be formed by passive tracers dragged by the flow and therefore the diffusion plays a significant role at certain scales. The retrieved power spectrum then differs from that expected for a direct measurement of the kinetic energy. Batchelor (1959) showed that the power spectrum for passive tracers follows a $k^{-1}$ law in the socalled viscous convective subrange between the "forcing" and the molecular diffusion spatial scales. This a simple and idealized approach to the turbulence laws, which assumes for example that there is enough separation between the forcing and dissipation scales for such trends to be observed.

Some previous works dealt with observations of this kind of power spectral laws, related to the kinetic energy (Earth and Jupiter) or cloud albedo and opacity (Earth, Venus and Jupiter). A summary of these results compared with our own values is presented in Table 5. Whether or not all of these results are linked with the turbulent regime of the atmosphere is still a major question that remains unanswered.

\section{[Table 5]}

All data sets follow a power-law trend with two slopes separated at a wavenumber turning point $k_{I}$ for both kinetic energy and cloud albedo/opacity power spectra. This happens even under extremely different atmospheric conditions: (a) slow 
rotator (Venus) versus rapid rotator (Earth and Jupiter); (b) presence of a surface (Venus and Earth) or not (Jupiter). This indicates that similar mechanisms may be operating in the wind and cloud fields. The Earth's kinetic energy power spectra show slopes of -3 and $-5 / 3$ (e.g. Nastrom et al. 1984; Nastrom and Gage 1985) in the opposite order as that predicted by Kraichnan (1967). This situation was later interpreted by Lilly (1989). For the rest of cases in Table 5, slopes and turning points differ and this is surely an indication of the different dynamical mechanisms operating in these atmospheres.

On Jupiter, we have measurements of the turbulent kinetic energy and the cloud albedo. The only measurement of the zonal kinetic energy power spectra were performed by Mitchell (1982) and Mitchell and Maxworthy (1985) using wind data from Voyager images. For jets between $40^{\circ} \mathrm{N}$ and $40^{\circ} \mathrm{S}$ they found the slope shown in Table 5 with the turning-point assumed to be located at $k=10$. On the other hand, Harrington et al. (1996b) studied the power spectra of Jupiter's cloud opacity spatial distribution at $5 \mu \mathrm{m}$. At this wavelength, the radiation coming from the interior of the planet is blocked by clouds somewhere between 2 and 5 bars pressure levels so they retrieved the power spectra of a deeper cloud.

Jupiter's results for larger scales range from $n_{1} \sim-0.7$ (Harrington et al. 1996b) to $n_{1} \sim-1.3$ (this work). On the other hand, results for smaller scales range from $n_{2} \sim-$ 2.5 (this work, excluding UV results to be commented later) to $n_{2} \sim-3.0$ (Mitchell 1982). Although they are based on different measurements (kinetic energy, cloud albedo and opacity) at different spatial resolutions and even at wavelengths ranging from the blue to the mid-infrared, all these figures are very close to each other. The main discrepancy between these works is found at the wavenumber turning point separating both regimes. Its value ranges from $k_{I}=10$ (assumed by Mitchell 1982) to our $k_{I} \sim 80$. If we inspect the wavenumber range $k=1-10$, we found an average value of the slope for 
wavenumbers $k=1-10, n_{0} \sim-1.8 \pm 0.5$ (for the near infrared and blue wavelengths). This slope is strongly affected by the presence of waves and shows a greater uncertainty. Conversely, if we consider the first slope $n_{l}$ to be extended down to wavenumber $k=1$ (instead of $k=10)$, the resulting value does not differ much from that presented so far, getting slightly higher than the values presented so far.

Within error bars, our slopes resemble the energy spectrum of 2D-turbulence (see for example figure 8.7 in Vallis 2006) with two sub-ranges separated at the "stirring" or "forcing" wavenumber. According to this interpretation, for scales $k<80$, the cloud brightness would be organized by the kinetic energy transfer favoring wave formation as evidenced by our periodicity analysis at some latitudes (Table 2). For scales $k>80$ the enstrophy transfer would be involved on the cloud brightness organization. This energy and enstrophy transfer is claimed to have been observed in Jupiter (Mitchell 1982), therefore pointing to a 2D-turbulence situation. A scheme comparing theoretical laws (2D and 3D regimes, kinetic energy and clouds) with observed Jovian spectra and different scales is drawn in Fig. 8., the available observational data point toward a turning point in the spectra between wavenumbers 10 and 80, which is within those expected for the Rhines and Rossby scales in Jupiter. These are two length scales that are relevant in the atmospheric dynamics and turbulence of a rapidly rotating planet. One is the Rhines scale $\left(L_{R}\right)$ (see e. g. Vallis 2006; Vasavada and Showman 2005; and references therein):

$$
L_{R}=\sqrt{u / \beta}
$$

where $u$ may be considered as the mean zonal wind speed $<u>\left(\sim 50-100 \mathrm{~ms}^{-1}\right)$ or as $u^{\prime}\left(\sim 5-10 \mathrm{~ms}^{-1}\right)$ the deviation of $u$ from the mean value. $\beta$ is the gradient of the Coriolis parameter $f, \beta=2 \Omega \cos \varphi / R(\varphi)$ being $\varphi$ the latitude, $\Omega$ is Jupiter's rotation period and $R(\varphi)$ the planetary radius at a given latitude. For $\varphi=45^{\circ}$, the Rhines scale for 
the above wind values ranges between $10^{3}$ to $10^{4} \mathrm{~km}$, corresponding to wavenumbers in the range $k=10-100$. The other relevant scale is the Rossby radius of deformation $\left(L_{D}\right)$ may also be calculated provided that we know the Brunt-Väisälä frequency $N$ and the height scale $H$ (Holton, 2004).

$$
L_{D}=N H / f
$$

Typical values for the troposphere at $\sim 45^{\circ}$ place it in the order of $\sim 1000 \mathrm{~km}(k \sim 100)$. This value agrees with that estimated by Read et al. (2006) from direct measurements of the temperature field. Therefore, these two scales fall within the range of wavenumbers encompassed by our power spectra analysis.

[Figure 8]

The behavior of the ultraviolet albedo power spectra is much more uncertain. On the one hand, the HST results differ from the Cassini ones. On the other hand, the transition is just the opposite than in the other two filters, changing from a steeper $(\sim$ 2.4 in HST, $\sim-1.5$ in Cassini) to a slighter $(\sim 0.3$, HST and $\sim-0.7$, Cassini $)$ slope. The HST case is not easily related to any theoretical or previous result and may be influenced by the lack of dynamic range in the observations. The Cassini values agree better with a passive tracer spectrum and this is consistent with the turning point being located at the same wavenumber than in the blue or infrared observations. At this level we find more static conditions and on a global scale and the dynamical regime is different to that on the main cloud deck. Except for a few features that give their imprint in the haze (e.g. large vortices as the GRS of the WOS), most details are bland, and the spectrum flattens.

In this work, we have investigated the behavior of the brightness power spectra finding a strong anisotropy between the zonal and meridional direction. There is still a long way to determine the relationship between the kinetic energy spectrum and the 
brightness power spectra. The only strict demonstration of such a relationship would consist in the direct measurement of the kinetic energy and comparison with the albedo power spectrum retrieved from the same high spatial resolution images. In fact, higher resolution would allow investigating higher wavenumbers and therefore it will allow checking other characteristic scales of the turbulent atmosphere of Jupiter.

\section{Acknowledgments}

This work has been funded by Spanish MEC AYA2006-07735 with FEDER support and Grupos UPV 15946/2004 and Grupos GV IT464-07. N. Barrado-Izagirre acknowledges a fellowship from UPV/EHU. This research made use of the public Cassini images available at NASA Planetary Data System. We acknowledge the Cassini-ISS team for their dedication in planning the observations. 


\section{References}

Arregi, J., Rojas, J.F., Sánchez-Lavega, A. and Morgado, A. 2006. Phase dispersion relation of the 5-micron Hot Spot wave from a long-term study of Jupiter in the visible. J. Geophys. Res., 111, E09010 (2006).

Barrado-Izagirre, N., Sánchez-Lavega A., Pérez-Hoyos S., Hueso R. 2008. Jupiter’s polar clouds and waves from Cassini and HST images: 1993-2006. Icarus, 194, 173185.

Barrey, R.F.T. 1984. A User's Guide to Voyager Image Processing. Atmospheric Physics Group, The Blanckett Laboratory, Imperial College, London.

Batchelor, G.K. 1959. Small scale variation of convected quantities like temperature in turbulent fluid. Part I: General discussion and the case of small conductivity. Journal of Fluid Mechanics, 5, $113-133$.

Beebe, R.F., Ingersoll, A.P., Hunt, G.E., Mitchell, J.L., Muller, J.P. 1980. Measurements of wind vectors, eddy momentum transports, and energy conversions in Jupiter's atmosphere from Voyager 1 images. Geophys. Res. Lett., 7, 1-4.

Brigham, E. O. 1988. The Fast Fourier Transform and its applications. Prentice-Hall, Englewood Cliffs, New Jersey. 
Cano, J.A. 1998. L.A.I.A.: Laboratorio de Análisis de Imágenes Astronómicas. Grup d'Estudis Astronòmics, Barcelona.

Cho, J. Y.-K. and Polvani, L.M. 1996. The emergence of jets and vortices in freely evolving, shallow-water turbulence on a sphere. Phys. Fluids, 8, $1531-1552$.

Del Genio, A.D., Barbara, J.M., Ferrier, J., Ingersoll, A.P., West, R.A., Vasavada, A.R., Spitale, J., Porco, C.C.2007. Saturn eddy momentum fluxes and convection: First estimates from Cassini images. Icarus, 189, 479-492.

Flasar, F.M. and 39 colleagues 2004. An intense stratospheric jet on Jupiter. Nature, $427,132-135$.

Fletcher, L.N., Irwin, P. G. J., Orton, G. S., Teanby, N. A., Achterberg, R. K., Bjoraker, G. L., Read, P. L., Simon-Miller, A. A., Howett, C., de Kok, R., Bowles, N., Calcutt, S. B., Hesman, B., Flasar, F. M. 2008. Temperature and Composition of Saturn's Polar Hot Spots and Hexagon. Science, 319, 5859, 79-82.

Galperin, B., Sukoriansky, S. and Huang, H.P. 2001. Universal $\mathrm{n}^{-5}$ spectrum of zonal flows on giant planets, Physics of Fluids, 13, 1545-1548.

Galperin, B., Nakano, H., Huang, H.P. and Sukoriansky, S. 2004. The ubiquitous zonal jets in the atmosperes of giant planets and Earth' oceans, Geophys. Res. Lett., 31, L13303. 
García-Melendo, E. and Sánchez-Lavega, A. 2001. A study of the stability of Jovian zonal winds from HST images: 1995-2000. Icarus, 152, 316 - 330.

Gierasch, P.J., Conrath, B.J. and Magalhães, J.A. 1986. Zonal mean properties of Jupiter's upper troposphere from voyager infrared observations. Icarus, 67, 456-483.

Harrington, J., Dowling, T.E. and Baron, R.L. 1996a. Jupiter's Tropospheric Thermal Emission: I. Observations and Techniques, Icarus, 124, $22-31$.

Harrington, J., Dowling, T.E. and. Baron, R.L 1996b. Jupiter's Tropospheric Thermal Emission: II. Power Analysis and Wave Search, Icarus, 124, 32 - 44.

Holton, J. R. 2004, An Introduction to Dynamic Meteorology; Academic Press, $4^{\text {th }}$ edition, $535 \mathrm{pp}$.

Horak, H.G. 1950. Diffuse reflection by planetary atmospheres. Astrophysical Journal, $112,445-463$.

Huang, H.-P., Galpering B. and Sukoriansky, S. 2001. Anisotropic spectra in twodimensional turbulence on the surface of a rotating sphere. Phys. Fluids, 13, 225.

Hueso, R., J. Legarreta, J. F. Rojas, J. Peralta, S. Pérez-Hoyos, T. del Río Gaztelurrutia and A. Sánchez-Lavega, The Planetary Laboratory for Image Analysis (PLIA), submitted. 
Ingersoll, A. P., Beebe, R. F., Mitchell, J. L., Garneau, G.W., Yagi, G. M., and Müller, J.-P. 1981. Interaction of eddies and mean zonal flow on Jupiter as inferred from Voyager 1 and 2 images. J. Geophys. Res., 86, 8733-8743.

Ingersoll, A.P., T.E. Dowling, P.J. Gierasch, G.S. Orton, P.L. Read, A. SánchezLavega, A.P. Showman, A.A. Simon-Miller, A.R. Vasavada 2004. Dynamics of Jupiter's atmosphere. In: Bagenal, F., Dowling, T.E., McKinnon, W.B. (Eds.), Jupiter: The Planet, Satellites, and Magnetosphere. Cambridge Univ. Press, pp. 105-128, chap. 6.

Kolmogorov, A. N. 1941b: The local structure in incompressible viscous fluid for very large Reynolds number (English translation 1991). Proc. Roy. Soc. London A, 434, 913.

Kraichnan, R. H. 1967: Inertial ranges in two-dimensional turbulence. Phys. Fluids, 10, 1417-1423.

Li, L., Ingersoll, A.P., Vasavada, A.R., Simon-Miller, A.A., Del Genio, A.D., Ewald, S.P. Porco, C.C and West, R.A.2006a. Vertical wind shear on Jupiter from Cassini images. J. Geophys. Res., 111, E04004.

Li, L., Ingersoll, A.P., Vasavada, A.R., Simon-Miller, A.A., Achterberg, R.K., Ewald, S.P., Dyudina, U.A., Porco, C.C., West, R.A., Flasar, F.M. 2006b. Waves in Jupiter's atmosphere observed by the Cassini ISS and CIRS instruments. Icarus, 185, 416-429. 
Lilly, D.K. 1989. Two-dimensional turbulence generated by energy sources at two scales. J. Atmos. Sci., 46, 2026-2030.

Lomb, N.R. 1976. Least-squares frequency analysis of unequally spaced data. Astrophysics and Space Sciences, 39, $447-462$.

Minnaert, M. 1941. The reciprocity principle in lunar photometry. The Astrophysical Journal, 93, $403-410$.

Mitchell, J.L. 1982. The nature of large-scale turbulence in the jovian atmosphere. Ph.D. Thesis. Jet Propulsion Laboratory, California Institute of Technology.

Mitchell, J.L. and T. Maxworthy 1985. Large-scale turbulence in the jovian atmosphere. In Turbulence Predictability in Geophisical Fluid Dynamics, pp. 226-240. Tipografia Compositori, Bologna, Italy.

Morgado, A., Sánchez-Lavega, A., Hueso, R., Rojas, J.F. 2005. PVOL: The Planetary Virtual Observatory \& Laboratory: An online database of the outer planet images. Bull. Am. Astron. Soc. 37, 652.

Nastrom, G.D., Jasperson, W. H. and Gage, K. S. 1984. Kinetic energy spectrum of large- and mesoscale atmospheric processes. Nature, 310, 36-38. 
Nastrom, G.D. and Gage, K.S. 1985. A climatology of atmospheric wavenumber spectra of winds and temperature observed by commercial aircraft. J. Atmos. Sci., 42, 950-960.

Peralta, J., Hueso, R., Sánchez-Lavega, A. 2007. Cloud brigthness distribution and turbulence in Venus using Galileo violet images. Icarus, 188, 305-314.

Pérez-Hoyos, S., Sánchez-Lavega, A., French, R.G., Rojas, J.F. 2005. Saturn's cloud structure and temporal evolution from ten years of Hubble Space Telescope Images (1994-2003). Icarus, 176, 155-174.

Porco, C., and 23 colleagues 2003. Cassini Imaging of Jupiter's Atmosphere, Satellites and Rings. Science, 299, $1541-1547$.

Porco, C., and 19 colleagues 2004. Cassini Imaging Science: Instrument Characteristics and Anticipated Scientific Investigations at Saturn. Space Sci. Rev. 115, 363 - 497.

Read, L.P., P.J. Gierasch, B.J. Conrath, A. Simon-Miller, T. Fouchet and Y.H. Yamazaki 2006. Mapping potential-vorticity dynamics on Jupiter. I: Zonal-mean circulation from Cassini and Voyager 1 data. Q.J.R. Meteorol. Soc., 132, 1577-1603.

Read, L.R., Yamazaki, Y.H., Lewis, S.R., Williams, P.D., Wordsworth R. and.MikiYamazaki, K 2007. Dynamics of convectively driven banded jets in the laboratory. J. Atmos. Sci., 64, 4031-4052. 
Rhines, P.B. 1975. Waves and turbulence on a beta-plane, J. Fluid Mech. 69, 417 443.

Salyk, C., Ingersoll, A.P., Lorre, J., Vasavada, A. and Del Genio, A.D. 2006. Interaction between eddies and mean flow in Jupiter's atmosphere: Analysis of Cassini imagining data. Icarus, $185,430-442$.

Sánchez-Lavega, A. and Gomez, J.M. 1996. The South Equatorial Belt of Jupiter, I: Its Life Cycle. Icarus,21, 1-17.

Sánchez-Lavega, A.,. Rojas, J.F. and Sada, P.V., 2000. Saturn's Zonal Winds at Cloud Level. Icarus, 147, 405-420.

Sánchez-Lavega, A., Orton, G.S., Morales, R., Lecacheux, J., Colas, F., Fisher, B., Fukumura-Sawada, P., Golischf, W., Griepf, D., Kaminski, C., Baines, K., Rages, K. and West, R. 2001. The merger of two giant anticyclones in the atmosphere of Jupiter. Icarus $149,491-495$.

Sánchez-Lavega, A., Hueso, R., Pérez-Hoyos, S., and Rojas, J.F. 2006. A strong vortex in Saturn's south pole. Icarus, 184, $524-531$.

Sánchez-Lavega A., G. S. Orton, R. Hueso, E. García-Melendo, S. Pérez-Hoyos, A. Simon-Miller, J. F. Rojas, J. M. Gómez, P. Yanamandra-Fisher, L. Fletcher, J. Joels, J. Kemerer, J. Hora, E. Karkoschka, I. de Pater, M. H. Wong, P. S. Marcus, N. PinillaAlonso, F. Carvalho, C. Go, D. Parker, M. Salway, M. Valimberti, A. Wesley and Z. 
Pujic 2008. Depth of a strong jovian jet from a planetary-scale disturbance driven by storms. Nature,451, $437-440$.

Scargle, J.D. 1982. Studies in astronomical time series analysis. II Statistical aspects of spectral analysis of unevenly spaced data. The Astrophysical Journal, 263, 835-835.

Scott, R.K. and Polvani, L.M. 2007. Forced-Dissipative Shallow-Water Turbulence on the Sphere and the Atmospheric Circulation of the Giant Planets. J. Atmos. Sci., 64, $3158-3176$.

Simon, A.A. 1999. The Structure and Temporal Stability of Jupiter's Zonal Winds: A Study of the North Tropical Region. Icarus, 141, 29-39.

Sromovsky, L.A., Revercomb H.E., Suomi V.E., Limaye S.S., Kraus R.J. 1982. Jovian winds from Voyager 2. Part II - Analysis of eddy transports. J. Atmos. Sci., 39, 14331445.

Sukoriansky, S., Galpering, B., and Dikovskaya, N. 2002. Universal Spectrum of TwoDimensional Turbulence on a Rotating Sphere and Some Basic Features of Atmospheric Circulation On Giant Planets. Phis. Rev. Lett. 89, 124501.

Travis, L.D., 1978. Nature of Atmospheric Dynamics on Venus from Power Spectrum Analysis of Mariner 10 Images, J. Atmos. Sci. 35, 1584-1595. 
Vallis, G.K. 2006. Atmospheric and Oceanic Fluid Dynamics. Cambridge University Press, Cambridge (UK).

Vasavada, A.R. and Showman, A.P. 2005. Jovian atmospheric dynamics: An update after Galileo and Cassini. Rep. Prog. Phys. 68, 1935-1996.

Vincent, M.B., and 18 colleagues, 2000. Jupiter's polar regions in the ultraviolet as imaged by HST/WFPC2: Auroral-aligned features and zonal motions. Icarus, 143, 205222.

West, R.A., Baines, K.H., Friedson, A.J., Banfield, D., Ragent, B. and Taylor F.W. 2004. Jovian clouds and hazes, in Jupiter: the planet, satellites and magnetosphere. Edited by F. Bagenal, T. E. Dowling, W. B. McKinnon. Cambridge planetary science, Vol. 1, Cambridge, UK.

Williams, G.P. 1978. Planetary circulations: 1. Barotropic representation of Jovian and Terrestrial turbulence. Journal of the Atmospheric Sciences 35, 1399 - 1426.

Williams, G.P. 1979. Planetary circulations: 2. The Jovian Quasi-Geostrophic regime. Journal of the Atmospheric Sciences 36, $932-968$. 
Table 1

HST-WFPC2 and Cassini-ISS filter description

\begin{tabular}{ccccc}
\hline Instrument & Date & Filter & $\boldsymbol{\lambda}_{\text {central }}$ & Width \\
\hline \multirow{2}{*}{ HST } & & F953N & $955 \mathrm{~nm}$ & $\sim 10 \mathrm{~nm}$ \\
WFPC2 & \multirow{2}{*}{$1995 / 10 / 05$} & F410M & $410 \mathrm{~nm}$ & $\sim 20 \mathrm{~nm}$ \\
& & F255W & $275 \mathrm{~nm}$ & $\sim 50 \mathrm{~nm}$ \\
\hline \multirow{2}{*}{ Cassini } & \multirow{2}{*}{$2000 / 11 / 14$} & CB3 & $938 \mathrm{~nm}$ & $\sim 10 \mathrm{~nm}$ \\
ISS & BL1 & $455 \mathrm{~nm}$ & $\sim 100 \mathrm{~nm}$ \\
& & UV1 & $258 \mathrm{~nm}$ & $\sim 50 \mathrm{~nm}$ \\
\hline
\end{tabular}


Table 2

Wave Location (near IR)

HST images (1995)

\begin{tabular}{ccc}
\hline Latitude range & Dominant Wavenumbers & Comments \\
\hline $16^{\circ} \mathrm{N}-20^{\circ} \mathrm{N}$ & $6,27,100$ & - \\
$13^{\circ} \mathrm{N}-15^{\circ} \mathrm{N}$ & $4,10,20$ & NEB-NTrZ interface \\
$4^{\circ} \mathrm{N}-8^{\circ} \mathrm{N}$ & 11,15 & Hot Spots \\
$0^{\circ}-3^{\circ} \mathrm{N}$ & 10,15 & Dark projections \\
$4^{\circ} \mathrm{S}-8^{\circ} \mathrm{S}$ & 8,52 & EZ-SEB boundary \\
$17^{\circ} \mathrm{S}-20^{\circ} \mathrm{S}$ & $8,18,37,55$ & STrZ-SEB boundary \& GRS wake \\
$32^{\circ} \mathrm{S}-38^{\circ} \mathrm{S}$ & $6-7$ & White Ovals \& Anticyclones \\
\hline & & \\
& Cassini images (2000) & \\
\hline \multicolumn{4}{c}{ Latitude range } & Dominant Wavenumbers & Comments \\
\hline $28^{\circ} \mathrm{N}-32^{\circ} \mathrm{N}$ & 17 & Dark small vortices \\
$20^{\circ} \mathrm{N}-26^{\circ} \mathrm{N}$ & 6,35 & NTB \\
$13^{\circ} \mathrm{N}-15^{\circ} \mathrm{N}$ & $4,10,24,52$ & NEB-NTrZ boundary \\
$2^{\circ} \mathrm{N}-8^{\circ} \mathrm{N}$ & 12 & Hot Spots \\
$1^{\circ} \mathrm{N}-1^{\circ} \mathrm{S}$ & 11,14 & Projections \\
$5^{\circ} \mathrm{S}-8^{\circ} \mathrm{S}$ & $9,50,75$ & EZ-SEB boundary \\
$15^{\circ} \mathrm{S}-22^{\circ} \mathrm{S}$ & $10,18,37,55$ & STrZ-SEB boundary \& GRS wake \\
$34^{\circ} \mathrm{S}-42^{\circ} \mathrm{S}$ & $6-7,51$ & White Ovals \& Anticyclones \\
\hline
\end{tabular}


Table 3

Power spectra slopes for meridional scans

\begin{tabular}{ccccc}
\hline Profile & Observations & Near Infrared & Blue & Ultraviolet \\
\hline \multirow{2}{*}{ Zonal wind } & $H S T$ & $-4.1 \pm 0.5$ & - & - \\
& Cassini & $-4.6 \pm 0.5$ & - & $-2.5 \pm 0.4$ \\
\hline \multirow{2}{*}{ Albedo } & $H S T$ & $-3.9 \pm 0.3$ & $-4.0 \pm 0.5$ & $-2.8 \pm 0.3$ \\
& Cassini & $-4.1 \pm 0.3$ & $-4.5 \pm 0.4$ & $-2.5 \pm 0.2$ \\
\hline
\end{tabular}


Table 4

Power spectra slopes for zonal scans

\begin{tabular}{|c|c|c|c|c|}
\hline \multirow[t]{2}{*}{ Wavelength } & \multirow[t]{2}{*}{$\begin{array}{l}\text { Integration } \\
\text { criterion }\end{array}$} & \multicolumn{2}{|c|}{$\begin{array}{c}\text { Power spectra slope } \\
\text { average }\end{array}$} & \multirow{2}{*}{$\begin{array}{c}\text { Wavenumber } \\
\text { turning point } \\
k_{I}\end{array}$} \\
\hline & & $<\mathbf{n}_{1}>$ & $<\mathbf{n}_{2}>$ & \\
\hline \multicolumn{5}{|c|}{1995 HST WFPC2 } \\
\hline \multirow{3}{*}{$953 \mathrm{~nm}$} & Jet Average & $-1.3 \pm 0.3$ & $-2.7 \pm 0.6$ & $80 \pm 18$ \\
\hline & Vorticity Average & $-1.3 \pm 0.3$ & $-2.7 \pm 0.6$ & $86 \pm 10$ \\
\hline & Brightness Average & $-1.3 \pm 0.4$ & $-2.6 \pm 0.6$ & $81 \pm 7$ \\
\hline \multirow{3}{*}{$410 \mathrm{~nm}$} & Jet Average & $-1.6 \pm 0.3$ & $-2.7 \pm 0.9$ & $84 \pm 14$ \\
\hline & Vorticity Average & $-1.6 \pm 0.4$ & $-2.7 \pm 0.7$ & $85 \pm 10$ \\
\hline & Brightness Average & $-1.6 \pm 0.4$ & $-3.1 \pm 1$ & $90 \pm 13$ \\
\hline \multirow{3}{*}{$255 \mathrm{~nm}$} & Jet Average & $-2.5 \pm 0.4$ & $-0.3 \pm 0.4$ & $85 \pm 12$ \\
\hline & Vorticity Average & $-2.4 \pm 0.5$ & $-0.3 \pm 0.5$ & $86 \pm 10$ \\
\hline & Brightness Average & $-2.3 \pm 0.5$ & $-0.4 \pm 0.3$ & $87 \pm 12$ \\
\hline \multicolumn{2}{|c|}{2000 Cassini ISS } & & +2 & \\
\hline \multirow{3}{*}{$938 \mathrm{~nm}$} & Jet Average & $-1.1 \pm 0.4$ & $-2.1 \pm 0.5$ & $74 \pm 12$ \\
\hline & Vorticity Average & $-1.2 \pm 0.4$ & $-2.3 \pm 0.7$ & $82 \pm 14$ \\
\hline & Brightness Average & $-1.2 \pm 0.4$ & $-2.2 \pm 0.5$ & $77 \pm 6$ \\
\hline \multirow{3}{*}{$455 \mathrm{~nm}$} & Jet Average & $-1.3 \pm 0.2$ & $-2.0 \pm 0.6$ & $84 \pm 14$ \\
\hline & Vorticity Average & $-1.3 \pm 0.4$ & $-2.0 \pm 0.5$ & $77 \pm 10$ \\
\hline & Brightness Average & $-1.3 \pm 0.4$ & $-2.0 \pm 0.5$ & $76 \pm 12$ \\
\hline \multirow{3}{*}{$258 \mathrm{~nm}$} & Jet Average & $-1.4 \pm 0.4$ & $-0.8 \pm 0.4$ & $84 \pm 11$ \\
\hline & Vorticity Average & $-1.5 \pm 0.4$ & $-0.7 \pm 0.4$ & $81 \pm 10$ \\
\hline & Brightness Average & $-1.5 \pm 0.3$ & $-0.7 \pm 0.3$ & $82 \pm 10$ \\
\hline
\end{tabular}


Table 5

Power spectra slopes and turning points for kinetic energy and cloud albedoopacity

\begin{tabular}{cccccc}
\hline Planet & $\mathbf{n}_{\mathbf{1}}$ & $\mathbf{n}_{\mathbf{2}}$ & $\mathbf{k}_{\mathbf{I}}$ & Comments & Reference \\
\hline Earth & -3 & $-5 / 3$ & 35 & Kinetic Energy & Nastrom et al. 1984 \\
\hline Venus & $-1.8 /-2.7$ & - & - & $\begin{array}{c}\text { Cloud Albedo } \\
(0.4 \mu \mathrm{m})\end{array}$ & Travis, 1978 \\
& -2.1 & -2.0 & 10 & $\begin{array}{c}\text { Cloud Albedo } \\
(0.4 \mu \mathrm{m})\end{array}$ & Peralta et al. 2007 \\
\hline Jupiter $^{(1)}$ & -1.2 & -3 & 10 & $\begin{array}{c}\text { Kinetic Energy } \\
\text { Cloud Opacity } \\
(5 \mu \mathrm{m})\end{array}$ & $\begin{array}{c}\text { Mitchell, 1982 } \\
\text { Harrington et al. } \\
1996\end{array}$ \\
& -0.7 & -2.9 & 26 & $\begin{array}{c}\text { Cloud Albedo } \\
(430 \mathrm{~nm}-950 \mathrm{~nm}) \\
\text { Cloud Albedo } \\
(260 \mathrm{~nm}, \text { Cassini })\end{array}$ & This Work \\
\hline (1) The cloud studies refer to three altitude levels: high $(250 \mathrm{~nm})$, mid $(430 \mathrm{~nm}-950 \mathrm{~nm})$ and deep $(5 \mu \mathrm{m})$. \\
\hline
\end{tabular}




\section{Figure captions}

Figure 1. Jupiter's planispheres and average filled contour map of Lomb-Scargle periodograms (up to wavenumber 18) for infrared (1a), blue (1b) and ultraviolet (1c). Top panels shows 1995 HST observations and the bottom ones, Cassini ISS observations. Note the different morphology and contrast of each case. Maps as a function of latitude and wavenumber show in lighter colours higher values of power (contours correspond to power values $0.01,12,50,75,100,150,200,300$ corresponding to a $\sim 100 \%$ false alarm probability for the first value, $10 \%$ f.a.p. for the second one, and lower than $1 \%$ f.a.p. for the rest).

Figure 2. Upper panel shows a stripe centred at $6.7^{\circ} \mathrm{N}$ latitude where the Hot Spots are located. The brightness profile is superimposed. On the bottom, we show the FFT of the profile in a linear scale using a Lomb-Scargle normalization (left) and the logarithmic brightness power spectrum (right), throwing dominant wavenumbers between 6 and 9 .

Figure 3. Upper panels show the relative intensity as a function of longitude and bottom panels include the retrieved power spectra and their slopes. (a) Sample power spectra at $54^{\circ} \mathrm{S}(1995-$ 953nm), (b) $21^{\circ} \mathrm{N}(1995-953 \mathrm{~nm})$ and (c) $45^{\circ} \mathrm{N}$ (2000-BL1), averaged in $5-6^{\circ}$ ranges around these latitudes.

Figure 4. Top panels show wind profiles and their power spectra: (a) Cassini 2000 by Porco et al. (2004) at $938 \mathrm{~nm}$ wavelength (CB3), (b) Cassini 2000 from ultraviolet images by Li et al. (2006a). The zonal energy power spectra plots are shown using $\Omega^{-1}$ and $R$ as time and longitude units. For comparison, the grey line shows the Galperin et al. (2001) $k^{-5}$ law with $C_{Z}=0.3$. Bottom panels show power spectra of the averaged brightness meridional profile 
for Cassini (2000) planispheres corresponding to (c) near infrared and (d) ultraviolet. Power spectra slopes are fitted in the range $k=20-90$, dashed line shows extrapolation to shorter and longer wavenumbers.

Figure 5. (a) Results for infrared 1995 (solid line) and 2000 (dashed line) planispheres. The three different averaging methods are shown; from top to bottom: wind direction, vorticity and brightness. First column shows: zonal wind profile by Garcia-Melendo et al. (2001), its corresponding meridional shear $d u / d y$ and the averaged meridional brightness profile. Middle column is the slope for lower wavenumbers $n_{1}$ and right column is for higher wavenumbers $n_{2}$. Vertical lines note the mean values for each wavenumber domain.

Figure 6. Same as Fig. 5 but for blue planispheres.

Figure 7. Same as Figs. 5 and 6 but for ultraviolet planispheres. Zonal wind profile here is taken from Li et al. (2006a).

Figure 8. Schematic comparison between turbulence theories and experimental results of this work. $\mathrm{Y}$ axis represents energy spectra and $\mathrm{X}$ the wavenumber, both are in logarithmical scale. Upper grey lines show the 2D-turbulent regime both for the kinetic energy spectrum (continuous line) and the passive tracer spectrum (dashed line). Bottom grey line is the same but for the 3D-turbulent regime. $k_{f}$ is the forcing scale, assumed to be at an arbitrary wavenumber. The three intermediate black curves show results from: (a) Mitchell (1982) and Mitchell and Maxworthy (1985), (b) Harrington et al. (1996b), (c) Cassini ultraviolet slopes (this work), and (d) blue and near-infrared slopes (this work), dashed line is used for the 
estimation of the lower wavenumbers slope. The shaded area depicts expected range of values for the Rhines and Rossby scales. 
Figure 1a

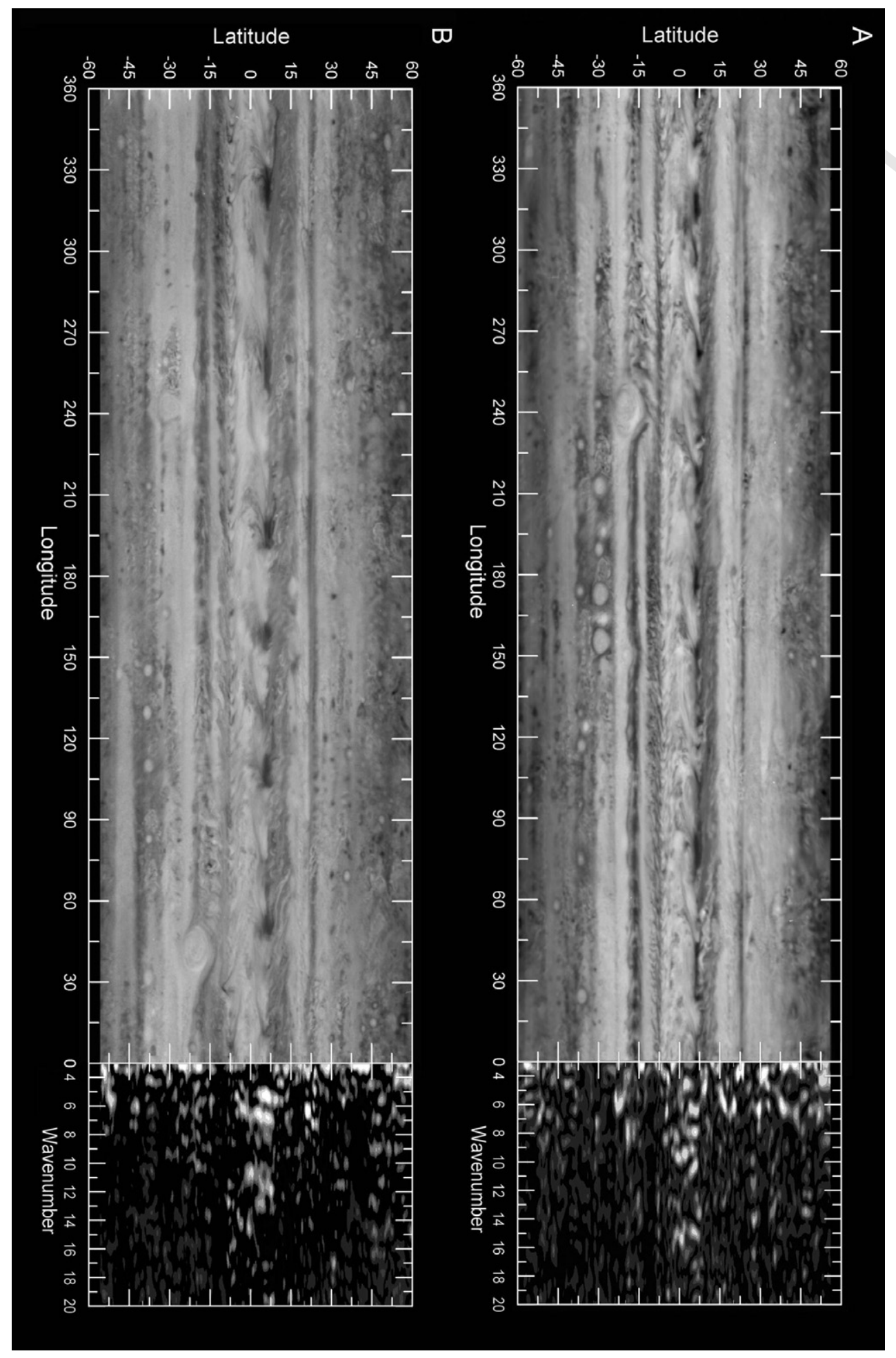


Figure 1b

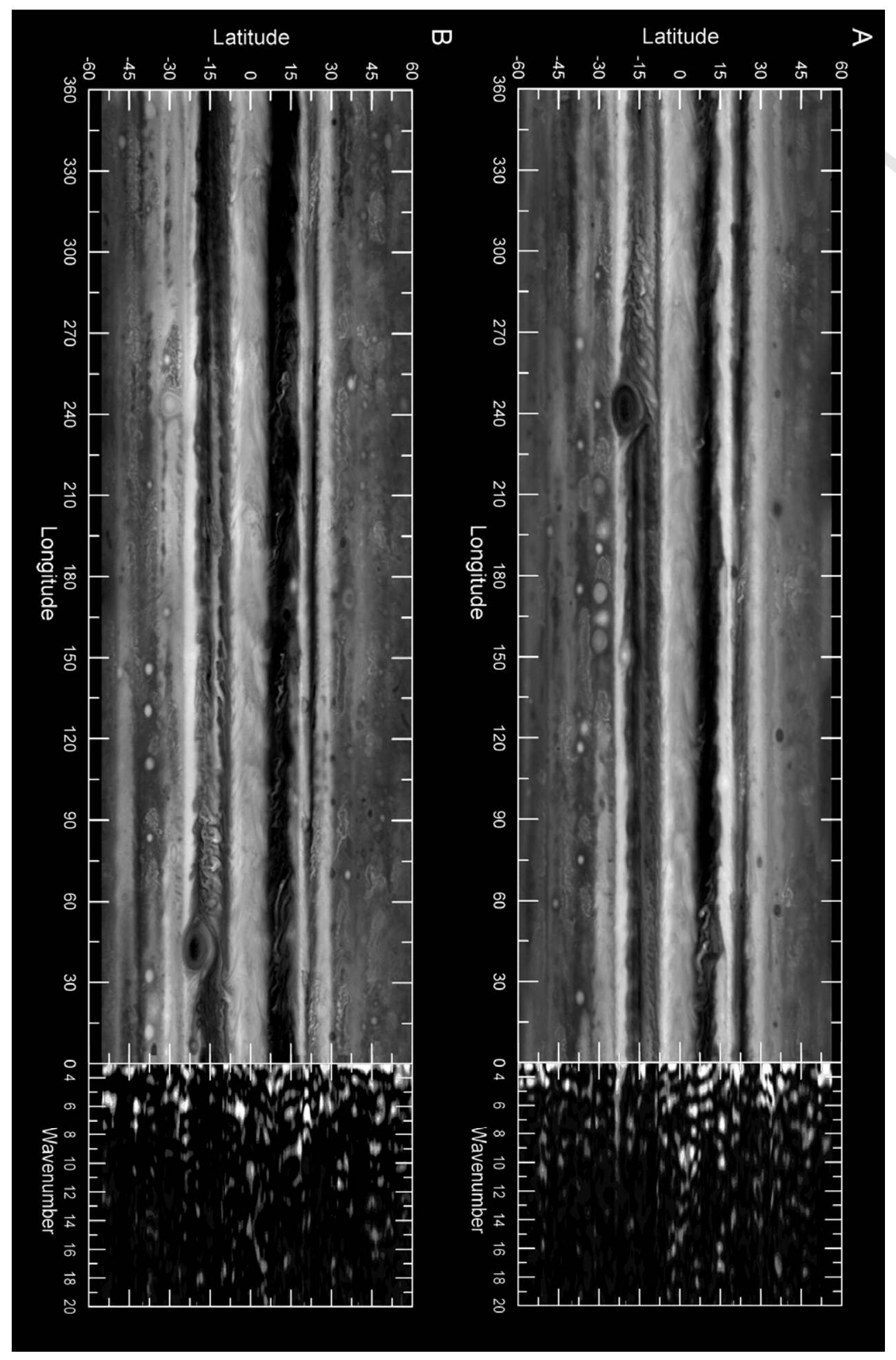


Figure 1c

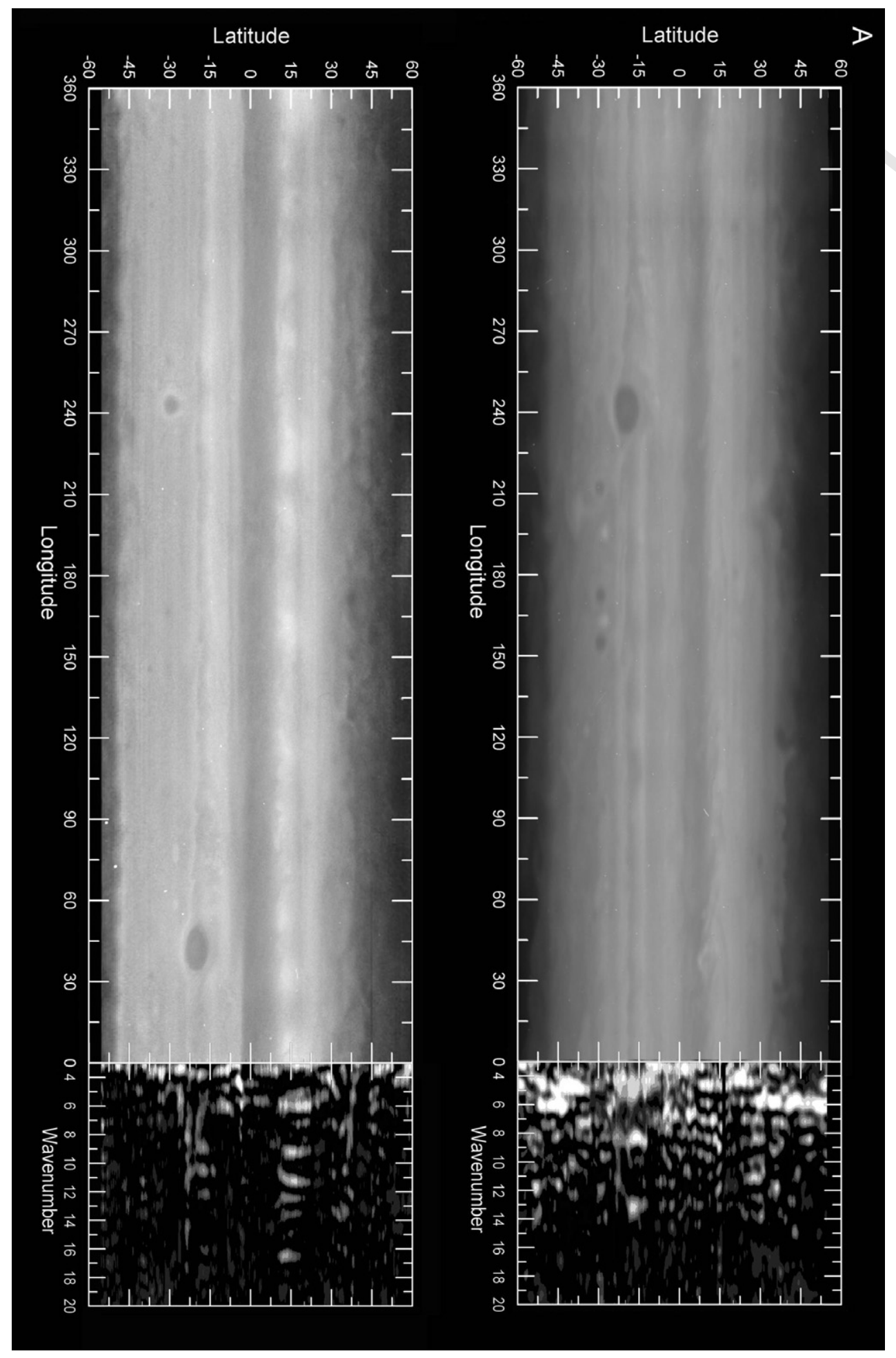


Figure 2
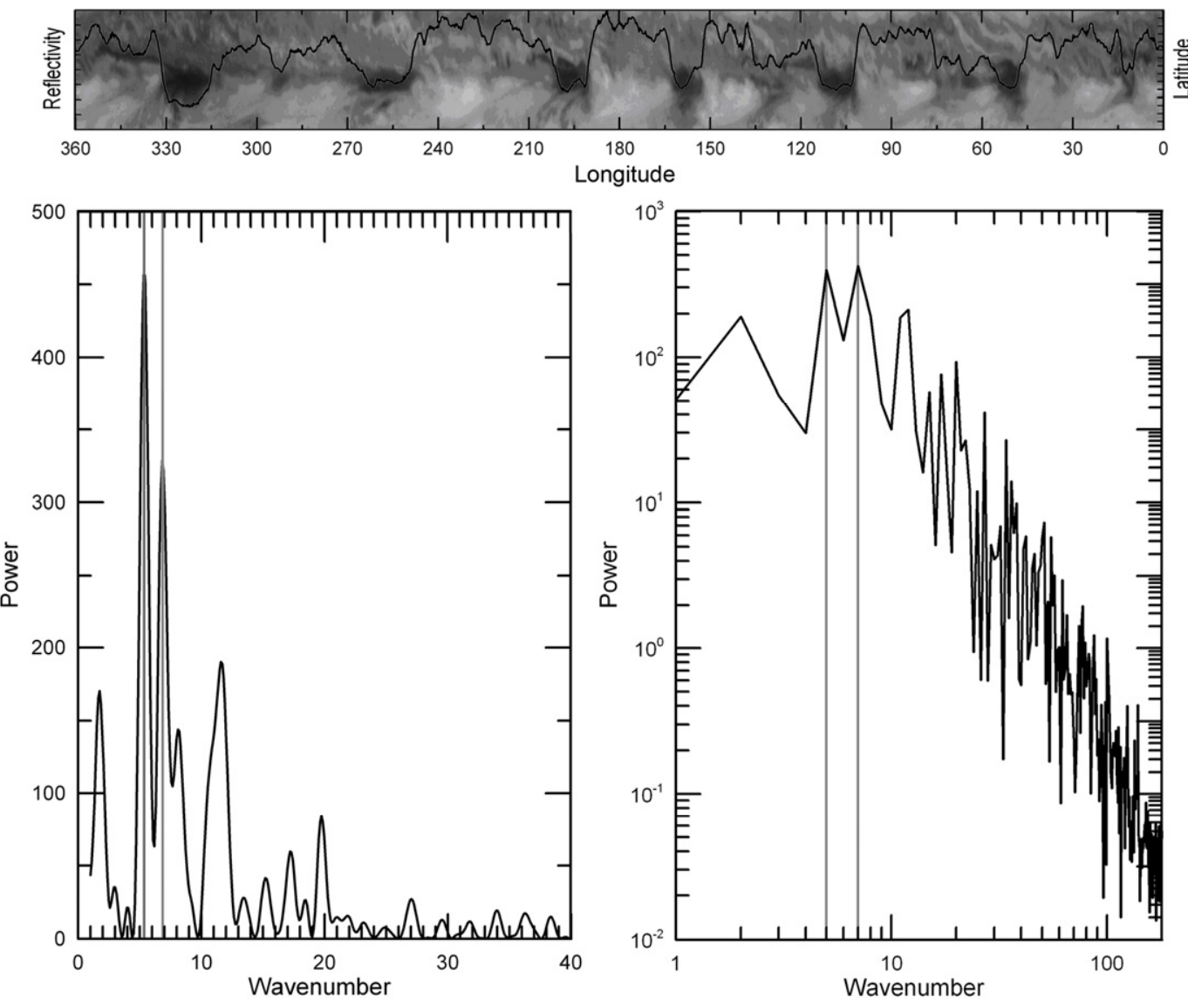
Figure 3

a)
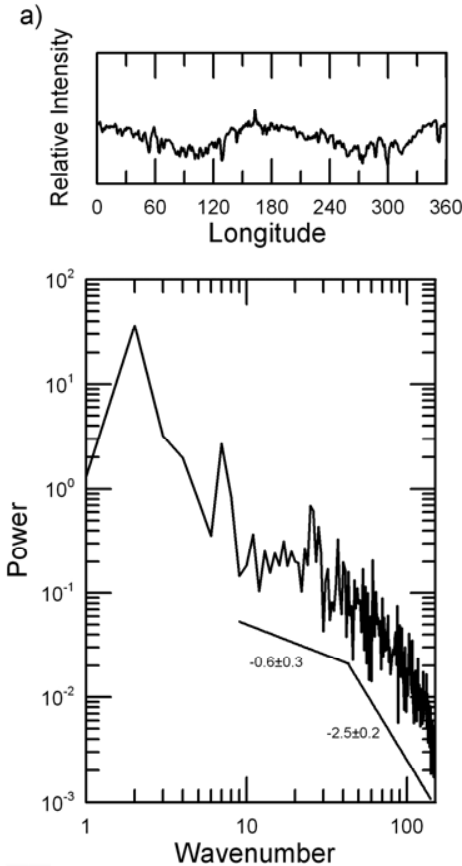

b)
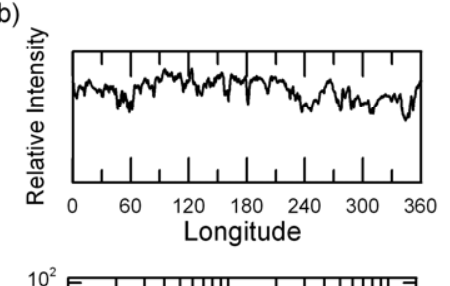

c)
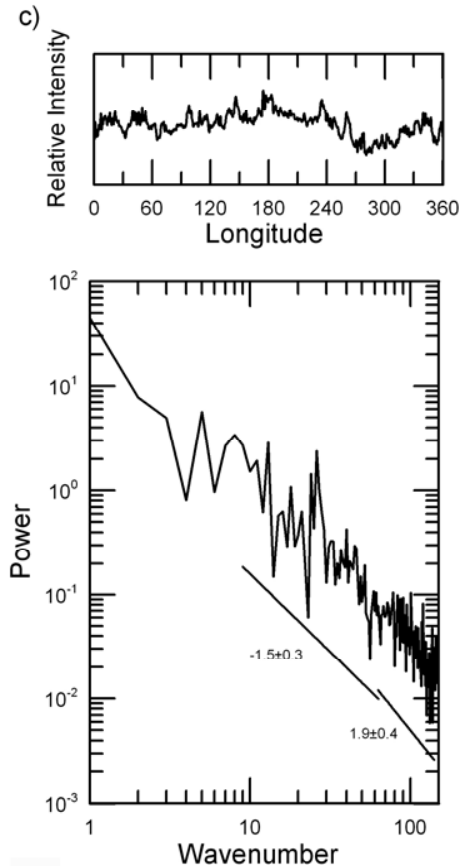
Figure 4
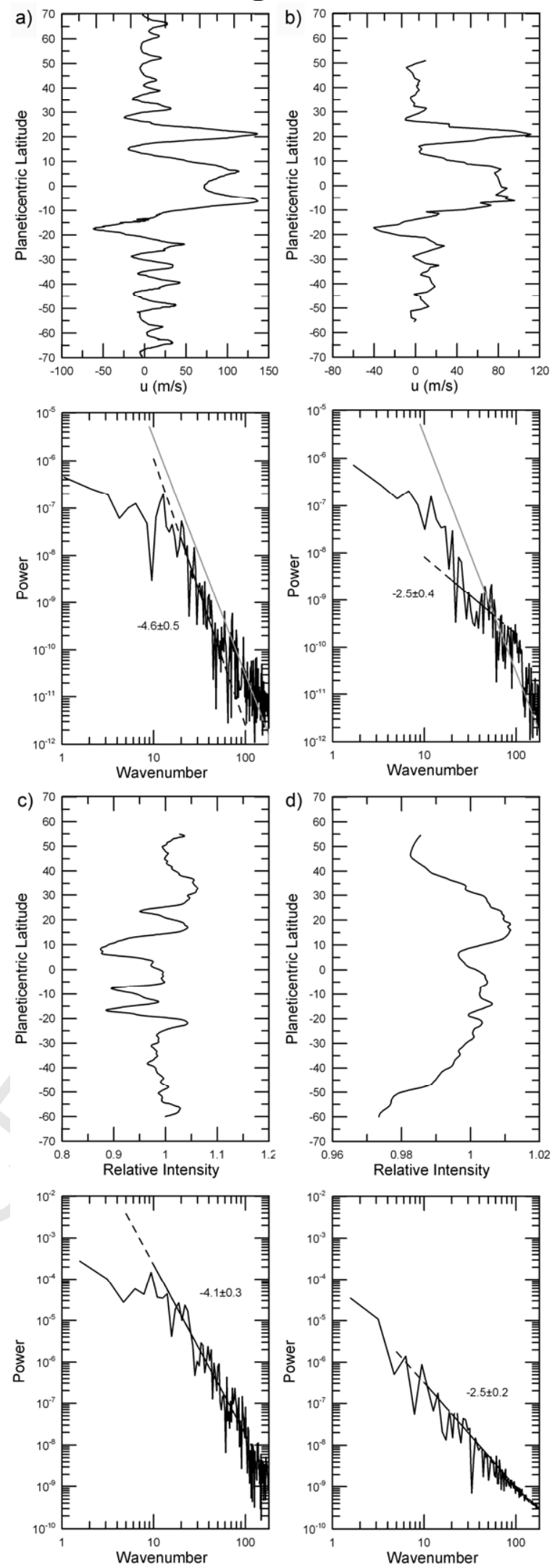
Figure 5
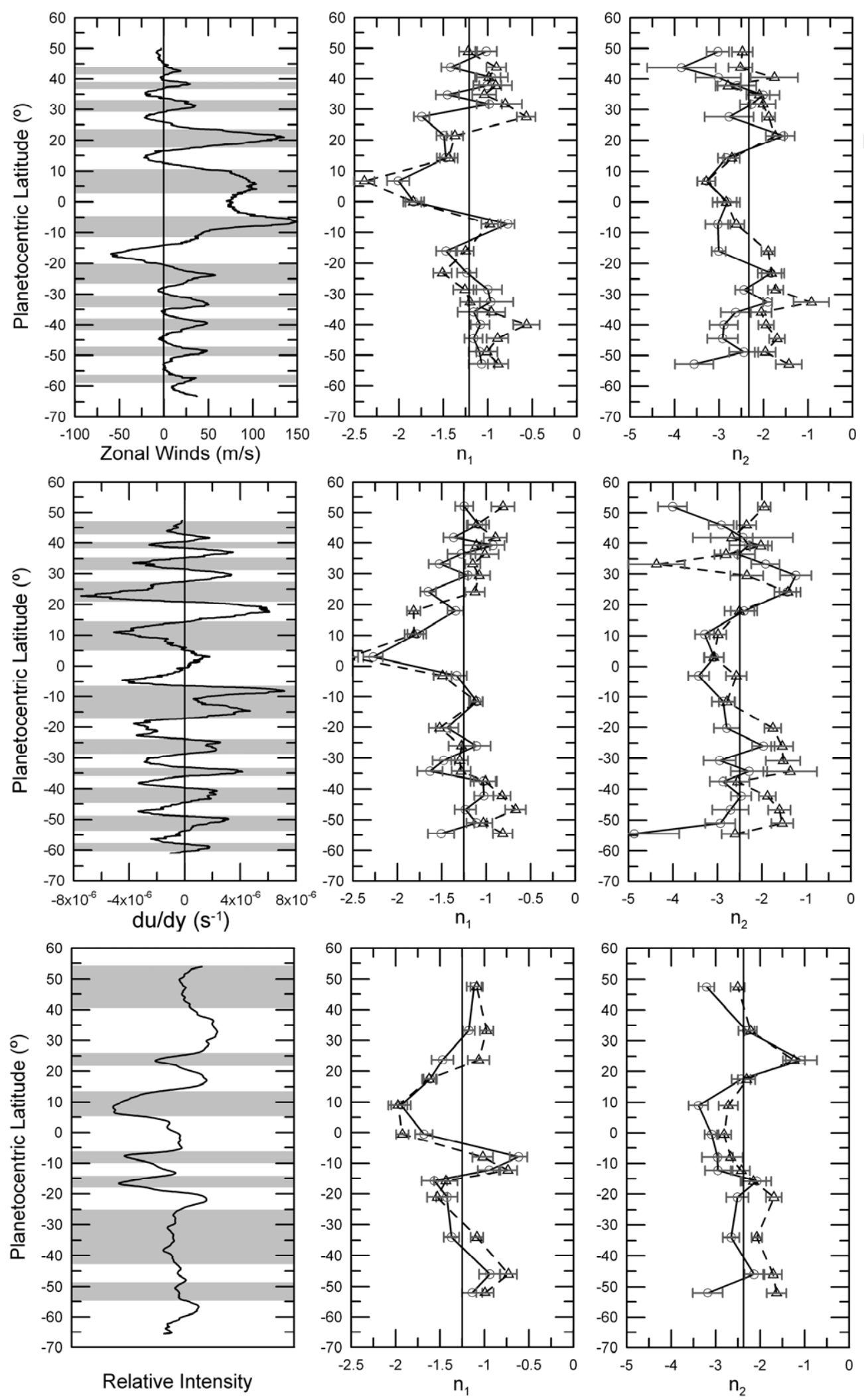


\section{Figure 6}
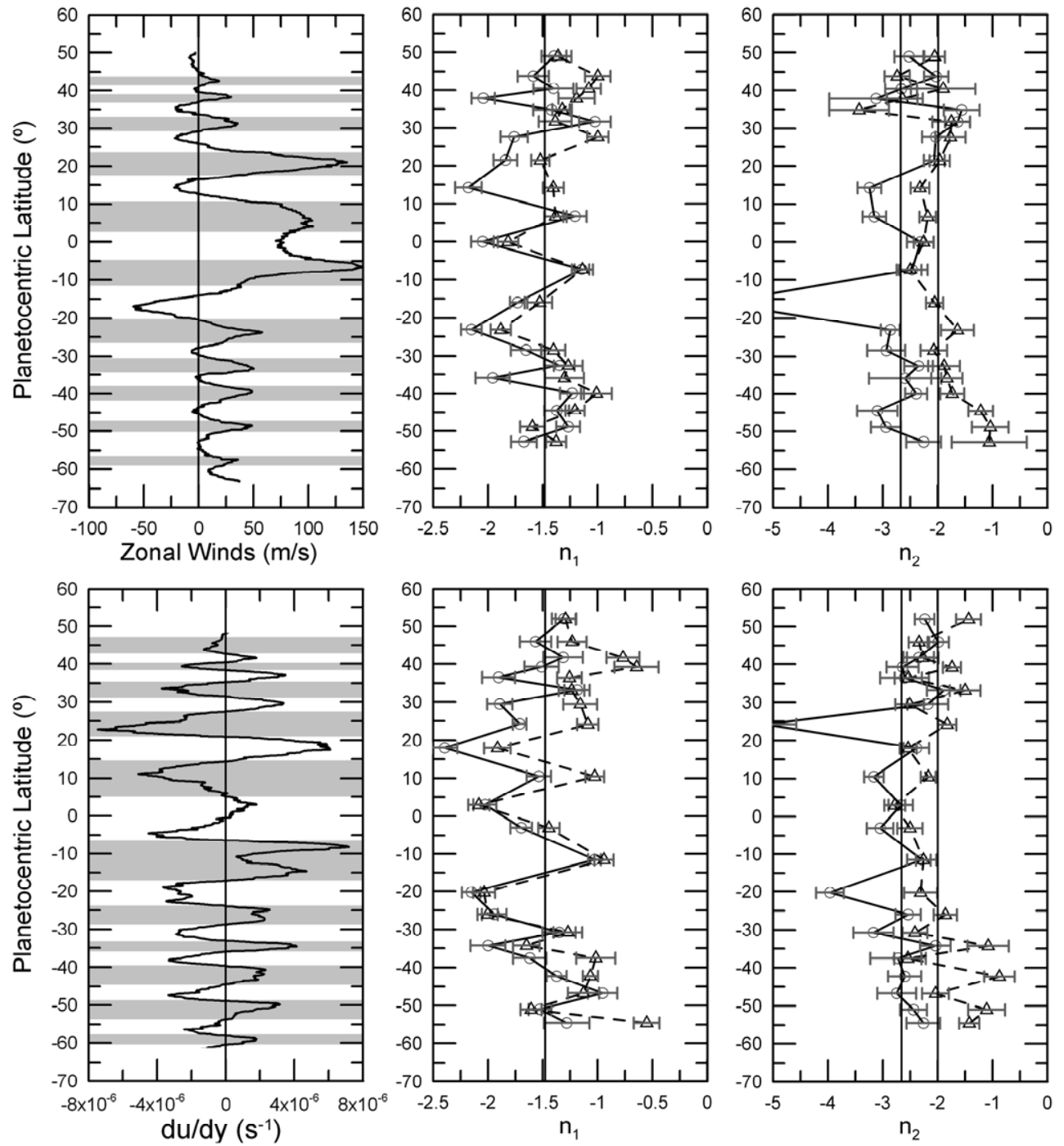

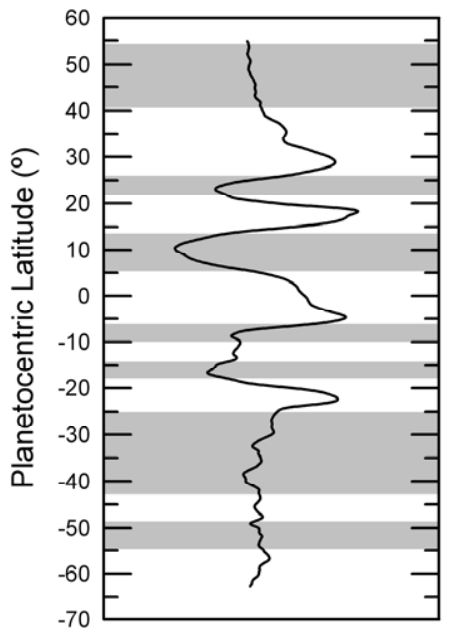

Relative Intensity
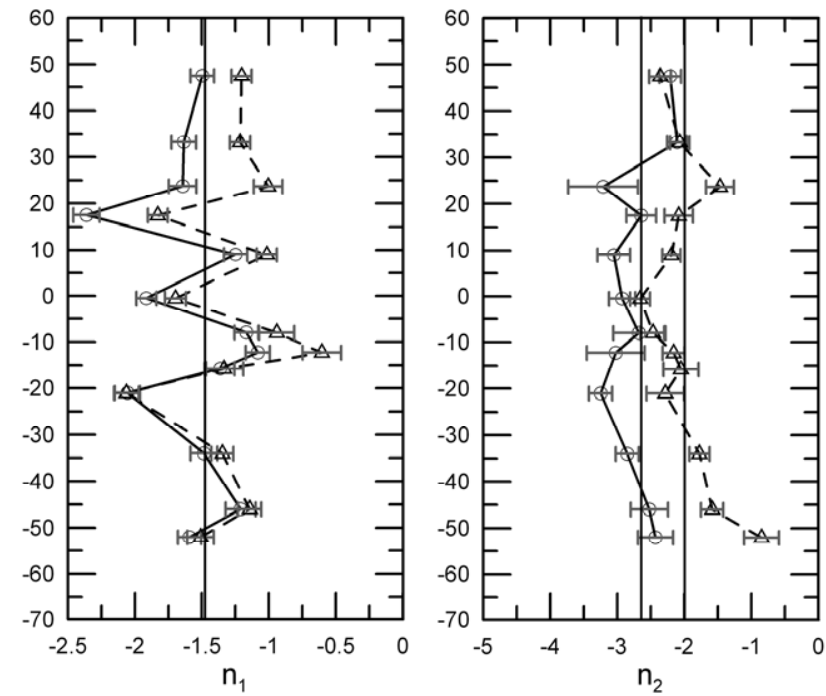
Figure 7
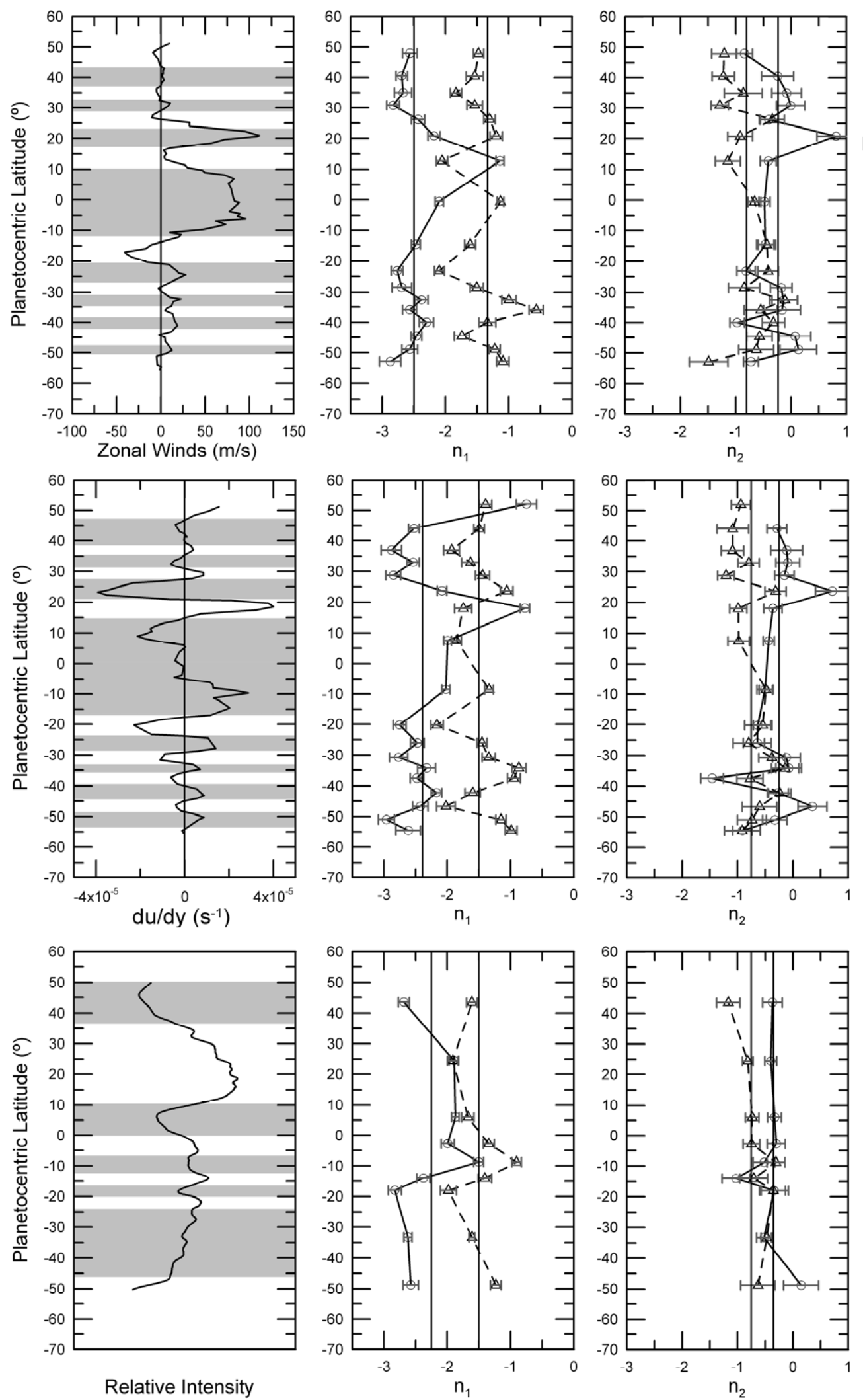
Figure 8

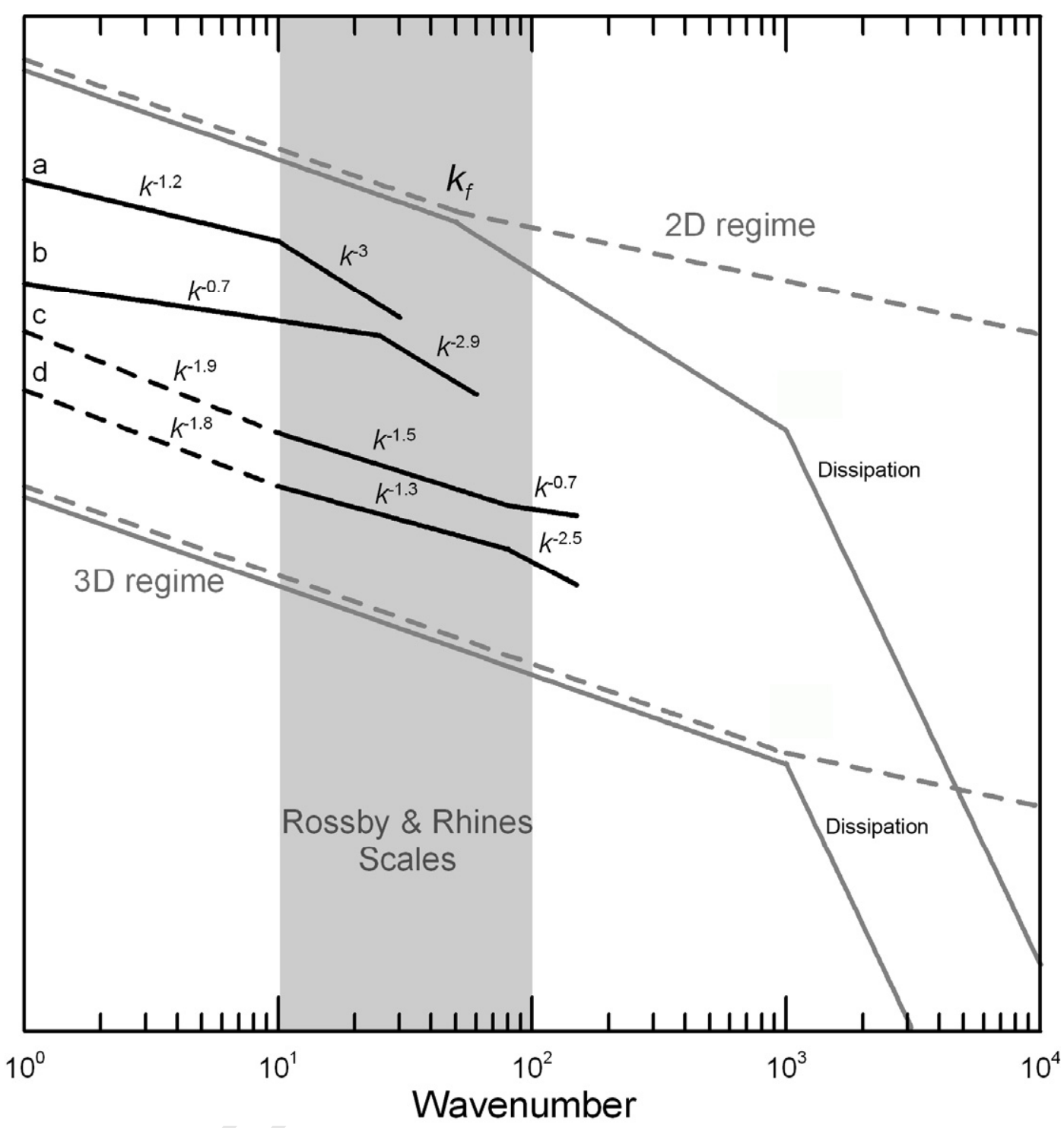

